\title{
Geometrically nonlinear dynamic analysis of functionally graded material plate excited by a moving load applying first-order shear deformation theory via generalized differential quadrature method
}

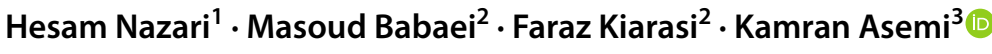

Received: 4 June 2021 / Accepted: 28 September 2021

Published online: 26 October 2021

(c) The Author(s) $2021 \quad$ OPEN

\begin{abstract}
In this study, we present a numerical solution for geometrically nonlinear dynamic analysis of functionally graded material rectangular plates excited to a moving load based on first-order shear deformation theory (FSDT) for the first time. To derive the governing equations of motion, Hamilton's principle, nonlinear Von Karman assumptions and FSDT are used. Finally, the governing equations of motion are solved by employing the generalized differential quadratic method as a numerical solution. Natural frequencies, dynamic bending behavior and stresses of the plate for linear and nonlinear type of geometrically strain-displacement relations and different factors, including the magnitude and velocity of moving load, length ratio, power law exponent and various edge conditions are obtained and compared.
\end{abstract}

Article highlights Developing generalized differential quadrature method (GDQM) solution based on FSDT for dynamic analysis of FGM plate excited by a moving load for the first time.

Comparison of linear and nonlinear dynamic response of plate by considering Von-Karman assumption.

Observing considerable difference between linear and nonlinear results

Keywords Functionally graded material - Nonlinear dynamic bending - First order shear deformation theory .

Generalized differential quadrature method $\cdot$ Moving load $\cdot$ Rectangular plate

\section{Introduction}

Nowadays, functionally graded materials (FGMs) are employed in various major sectors of industries due to fantastic mechanical property. An important application of FGMs is bridges that are extensively applied in railway industry [1-5]. Therefore, it is essential to denote the dynamic characteristics of these type of structures under moving loads. Many researches have been conducted about the behavior of these structure under moving loads that some of them are mentioned here. Yang et al. [6] analytically investigated natural frequencies and dynamic responses of cracked inhomogeneous beams under an axial load and a moving force based on Euler-Bernoulli beams. Malekzadeh and Monajjemzadeh [7] studied linear dynamic response of rectangular plates made of FGMs in the presence of temperature under moving force based on FSDT and using finite element method (FEM) [8]. In another study, they employed classical plate theory (CLPT) and FEM in conjunction with Newmark procedure to study

Kamran Asemi, K.asemi@iau-tnb.ac.ir; Hesam Nazari, hesamnazari1994@gmail.com; Masoud Babaei, masoudbabaei@eyc.ac.ir; Faraz Kiarasi, f.kiarasi@eyc.ac.ir | ${ }^{1}$ Department of Mechanical Engineering, Science and Research Branch, Islamic Azad University, Tehran, Iran. ${ }^{2}$ Department of Mechanical Engineering, University of Eyvanekey, Eyvanekey, Semnan, Iran. ${ }^{3}$ Department of Mechanical Engineering, Faculty of Engineering, North Tehran Branch, Islamic Azad University, Tehran, Iran. 
the nonlinear behavior of FGM plates subjected to a moving force. Dynamic characteristics of cylinders made of an FGMs excited by a moving force were presented by Sofiyev [9]. Chen et al. [10] investigated geometrically nonlinear dynamic response of tubes made of FGM under moving force by employing refined beam model by employing Galarkin method. Yas and Heshmati [11] surveyed linear dynamic behavior of beams reinforced with single-wall carbon nanotubes (SWCNT) under moving force applying Timoshenko and Euler-Bernoulli beam theories. Based on lagrangian and Newmark- $\beta$ methods, large deflection dynamic response of a Timoshenko FGM beam excited by a moving harmonic force was presented by Şimşek [12]. Ebrahimzadeh Hassanabadi et al. [13] investigated linear dynamic behavior of plates under moving mass and by applying CLPT and also employing a benchmark solution. Esen [14] presented a novel numerical FEM solution to study linear free vibration response of plates excited by moving forces by applying CLPT. Assie et al. [15] applied Ritz procedure in conjunction with Newmark method to study linear natural frequencies of perforated Timoshenko beam under a moving force. Uzzal et al. [16] performed an investigation about linear dynamic characteristics of beams supported on Pasternak elastic foundation excited by a moving force and masses. Nguyen [17] investigated linear natural frequencies of two dimensional FGM beams excited by a moving force based on Timoshenko theory and by employing FEM. Kiani carried out investigation about linear dynamics of composite cylindrical [18] -conical [19] panels made of functionally graded carbon nanotube (FG-CNT) excited by a moving load using FSDT and applying Ritz procedure. In another investigation, Kiani [20] conducted the effects of graphene platelets (GPLs) reinforcement on the linear dynamic bending behavior of rectangular plates excited by a moving force applying FSDT and Ritz methods. Sorrentino and Catania [21] developed a semi-analytical procedure for linear dynamic bending behavior of plates under a moving force by employing CPLT. Shirmohammadi et al. [22] performed linear dynamic analysis of rectangular plates excited by moving forces by employing spectral finite strip approach based on CLPT theory. Eyvazian [23] investigated dynamic behavior of cylindrical shells reinforced by GPLs excited by a moving harmonic force applying FSDT by considering various boundary conditions. Luong et al. [24] used moving element technique based on FSDT to survey linear dynamic response of FGM rectangular plates supporting on Pasternak elastic foundation excited by moving force. Based on FSDT and by employing dynamic relaxation method, linear dynamic behavior of two directionally FGM porous rectangular plates excited by a moving load was presented by Esmaeilzadeh and Kadkhodayan [25]. Akgün et al. [26] employed GDQ method to investigate non-linear transient response of porous functionally graded truncated conical panels based on FSDT theory. Akgün and Kurtaran [27] applied GDQ method to study large displacement transient analysis super-elliptic shells made of FGM based on FSDT theory. Nonlinear dynamic and vibration analysis of piezoelectric eccentrically stiffened FGM plates based on first-order shear deformation theory in thermal environment was presented by Duc et al. [28]. Duc et al. [29] studied transient behaviour of functionally graded double curved shallow shells based on classical shell theory in thermal environment by using Galerkin method. Based on higher shear deformation theory and by using Galerkin method, Nonlinear dynamic response and vibration of functionally graded carbon nanotube reinforced composite double curved shallow shells subjected to blast load and temperature were presented by Duc et al. [30]. Duc et al. [31] employed FSDT theory and Galerkin method to investigate nonlinear dynamic and vibration analyses of shear deformable eccentrically stiffened S-FGM cylindrical panels resting on elastic foundations. Nonlinear static and dynamic stability of functionally graded plates and shells was presented by Duc et al. [32]. Cong et al. [33] employed third order shear deformation theory (TSDT) and Galerkin method to investigate nonlinear dynamic behavior of sandwich auxetic double curves shallow shells.

Based on literature review, most of studies concentrated on linear dynamic characteristics of beam and plates excited by a moving force by applying FEM as numerical method. Therefore, nonlinear dynamic analysis of FGM plates applying GDQM has not been investigated. In the present investigation, a numerical procedure by using GDQM is conducted for analyzing nonlinear dynamic behavior of FGM plates excited by moving loads employing Hamilton's principle, Von Karman assumptions and FSDT theory. Transient deflection and stresses of FGM plate have been investigated for different material gradient index, aspect ratio, magnitude and velocity of moving load and various boundary conditions. Also, by excluding nonlinear terms of strain and displacements relations, linear and nonlinear dynamic response of plate are compared. In Sect. 2, the displacement fields are assumed based on FSDT. After that, we obtain the strains based on displacement fields. Also, the stress components are replaced with strains according to Hooke's law. Then, the Hamilton's principle is employed to obtain the governing equations of motion and boundary conditions by applying calculus of variation. Finally, by applying GDQ method, the governing equations are solved. In Sect. 3 , after the validation of the present work by previous studies, linear and nonlinear results are presented for natural frequencies and dynamic bending of FGM plate by considering different factors, including the magnitude and velocity of moving 


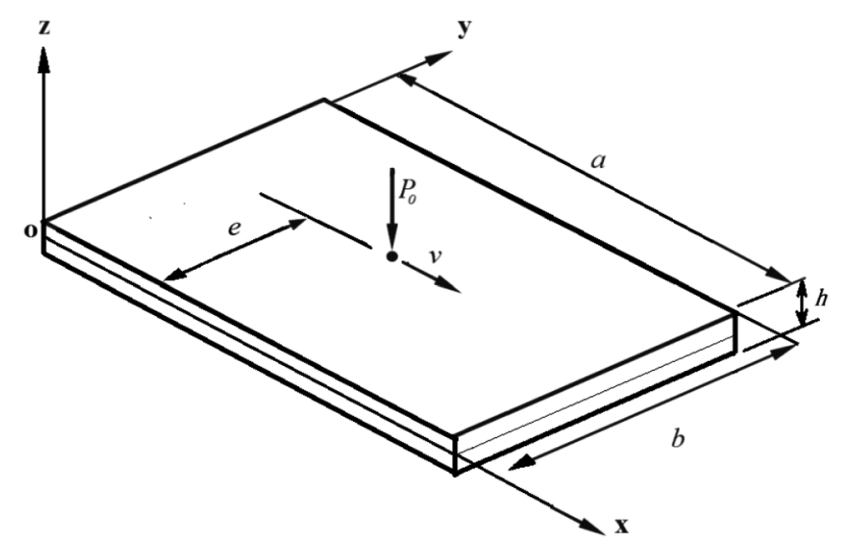

Fig. 1 Schematic of FGM plate excited by a moving force

load, length ratio, power law exponent and various edge conditions.

\section{Mathematical formulation}

\subsection{Geometry and material property}

Consider an FGM rectangular plate excited by a moving load, as shown in Fig. 1. The structure is constructed of a ceramic-metal mixture, and the mechanical properties are determined to change smoothly along the $z$ direction. FSDT theory is applied according to the thickness of plate. Hence, the displacement fields are assumed as [34]:

$$
\begin{aligned}
& u_{x}(x, y, z, t)=u_{0}(x, y, t)+z \psi_{x}(x, y, t) \\
& u_{y}(x, y, z, t)=v_{0}(x, y, t)+z \psi_{y}(x, y, t) \\
& u_{z}(x, y, z, t)=w_{0}(x, y, t)
\end{aligned}
$$

The plate is made of a metal-ceramic FGM. The volume fraction of the metal material is supposed to change along the $z$ direction as follows:

$V_{m}=\left(\frac{z}{h}+\frac{1}{2}\right)^{n}$

The upper and lower faces of the structure are made of metal and ceramic, respectively. Also, $n$ is the positivedefinite power law exponent. Therefore, the mechanical properties of the structure such as the modulus of elasticity and mass density are defined as:

$$
E=E_{c}+\left(E_{m}-E_{c}\right)\left(\frac{z}{h}+\frac{1}{2}\right)^{n}
$$

$$
\rho=\rho_{c}+\left(\rho_{m}-\rho_{c}\right)\left(\frac{z}{h}+\frac{1}{2}\right)^{n}
$$

E and $\rho$ are the elasticity modules and density of FGM structure. Also, indices $\mathrm{c}$ and $\mathrm{m}$ denote the material properties of ceramic and metal. The Poisson's ratio is supposed to be constant.

In Eq. (1), u, v, w, are displacement fields across the $x, y$ and $z$ axes, respectively. Besides, each axis with 0 subscript denotes the mid-plane displacement field. Also, $\psi_{x}$ and $\psi_{y}$ are normal transverse rotations around $\mathrm{y}$ and $\mathrm{x}$ axes, respectively. Von-Karman strain-displacement relationships are defined as:

$$
\begin{aligned}
& \varepsilon_{x x}=\frac{1}{2}\left(\frac{\partial u_{x}}{\partial x}+\frac{\partial u_{x}}{\partial x}+\frac{\partial u_{z}}{\partial x} \frac{\partial u_{z}}{\partial x}\right)=\frac{\partial u_{0}}{\partial x}+z \frac{\partial \psi_{x}}{\partial x}+\frac{1}{2}\left(\frac{\partial w_{0}}{\partial x}\right)^{2} \\
& \varepsilon_{y y}=\frac{1}{2}\left(\frac{\partial u_{y}}{\partial y}+\frac{\partial u_{y}}{\partial y}+\frac{\partial u_{z}}{\partial y} \frac{\partial u_{z}}{\partial y}\right)=\frac{\partial v_{0}}{\partial y}+z \frac{\partial \psi_{y}}{\partial y}+\frac{1}{2}\left(\frac{\partial w_{0}}{\partial y}\right)^{2} \\
& \gamma_{x y}=\left(\frac{\partial u_{x}}{\partial y}+\frac{\partial u_{y}}{\partial x}+\frac{\partial u_{z}}{\partial x} \frac{\partial u_{z}}{\partial y}\right)=\frac{\partial u_{0}}{\partial y}+\frac{\partial v_{0}}{\partial x}+z\left(\frac{\partial \psi_{x}}{\partial y}+\frac{\partial \psi_{y}}{\partial x}\right)+\frac{\partial w_{0}}{\partial x} \frac{\partial w_{0}}{\partial y} \\
& \gamma_{x z}=\left(\frac{\partial u_{x}}{\partial z}+\frac{\partial u_{z}}{\partial x}+\frac{\partial u_{z}}{\partial x} \frac{\partial u_{z}}{\partial z}\right)=\psi_{x}+\frac{\partial w_{0}}{\partial x} \\
& \gamma_{y z}=\left(\frac{\partial u_{y}}{\partial z}+\frac{\partial u_{z}}{\partial y}+\frac{\partial u_{z}}{\partial y} \frac{\partial u_{z}}{\partial z}\right)=\psi_{y}+\frac{\partial w_{0}}{\partial y}
\end{aligned}
$$




\subsection{Governing equation of motion}

The Relationship between stress and strain of FGM rectangular plate based on FSDT is as the following: correction factor. The strain energy of structure is as the following:

$\delta U=\iiint_{V}\left(\sigma_{x x} \delta \varepsilon_{x x}+\sigma_{y y} \delta \varepsilon_{y y}+\tau_{x y} \delta \gamma_{x y}+\tau_{x z} \delta \gamma_{x z}+\tau_{y z} \delta \gamma_{y z}\right) d V$

By replacing strain component, we have:

The resultants of normal and shear forces and moments are obtained by integrating the stress field along the thickness direction:

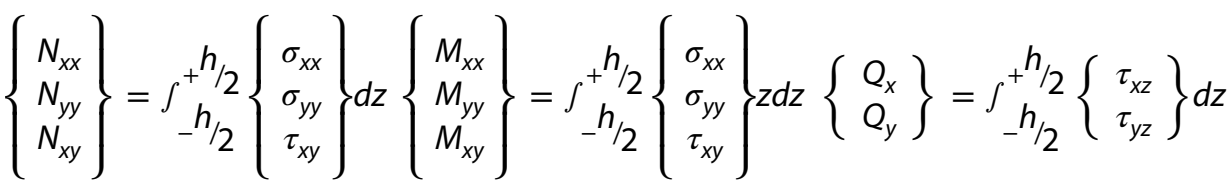

We can rewrite Eq. (6) by using Eq. (7) as following:

$\delta U=\iint_{A}\left(\begin{array}{l}N_{x x} \delta\left(\frac{\partial u_{0}}{\partial x}\right)+M_{x x} \delta\left(\frac{\partial \psi_{x}}{\partial x}\right)+\frac{N_{x x}}{2} \delta\left(\frac{\partial w_{0}}{\partial x}\right)^{2}+N_{y y} \delta\left(\frac{\partial v_{0}}{\partial y}\right)+M_{y y} \delta\left(\frac{\partial \psi_{y}}{\partial y}\right) \\ +\frac{N_{y y}}{2} \delta\left(\frac{\partial w_{0}}{\partial y}\right)^{2}+N_{x y} \delta\left(\frac{\partial u_{0}}{\partial y}\right)+N_{x y} \delta\left(\frac{\partial v_{0}}{\partial x}\right)+M_{x y} \delta\left(\frac{\partial \psi_{x}}{\partial y}+\frac{\partial \psi_{y}}{\partial x}\right) \\ +N_{x y} \delta\left(\frac{\partial w_{0}}{\partial x} \frac{\partial w_{0}}{\partial y}\right)+Q_{x} \delta\left(\psi_{x}+\frac{\partial w_{0}}{\partial x}\right)+Q_{y} \delta\left(\psi_{y}+\frac{\partial w_{0}}{\partial y}\right)\end{array}\right) d A$

$$
\begin{aligned}
& \sigma_{x x}=\frac{E}{1-v^{2}}\left(\varepsilon_{x x}+v \varepsilon_{y y}\right)=\frac{E}{1-v^{2}}\left[\frac{\partial u_{0}}{\partial x}+v \frac{\partial v_{0}}{\partial y}+\frac{1}{2}\left[\left(\frac{\partial w_{0}}{\partial x}\right)^{2}+v\left(\frac{\partial w_{0}}{\partial y}\right)^{2}\right]+z\left(\frac{\partial \psi_{x}}{\partial x}+v \frac{\partial \psi_{y}}{\partial y}\right)\right] \\
& \sigma_{y y}=\frac{E}{1-v^{2}}\left(v \varepsilon_{x x}+\varepsilon_{y y}\right)=\frac{E}{1-v^{2}}\left[v \frac{\partial u_{0}}{\partial x}+\frac{\partial v_{0}}{\partial y}+\frac{1}{2}\left[v\left(\frac{\partial w_{0}}{\partial x}\right)^{2}+\left(\frac{\partial w_{0}}{\partial y}\right)^{2}\right]+z\left(v \frac{\partial \psi_{x}}{\partial x}+\frac{\partial \psi_{y}}{\partial y}\right)\right] \\
& \tau_{x y}=\frac{E}{2(1+v)} \gamma_{x y}=\frac{E}{2(1+v)}\left[\frac{\partial u_{0}}{\partial y}+\frac{\partial v_{0}}{\partial x}+z\left(\frac{\partial \psi_{x}}{\partial y}+\frac{\partial \psi_{y}}{\partial x}\right)+\frac{\partial w_{0}}{\partial x} \frac{\partial w_{0}}{\partial y}\right] \\
& \tau_{x z}=\kappa \frac{E}{2(1+v)} \gamma_{x z}=\kappa \frac{E}{2(1+v)}\left(\psi_{x}+\frac{\partial w_{0}}{\partial x}\right) \\
& \tau_{y z}=\kappa \frac{E}{2(1+v)} \gamma_{y z}=\kappa \frac{E}{2(1+v)}\left(\psi_{y}+\frac{\partial w_{0}}{\partial y}\right)
\end{aligned}
$$

where $\mathrm{E}$ and $\mathrm{v}$ are the elasticity modules and Poisson's ratio, respectively. In addition, $K=\frac{\pi^{2}}{12}[35]$ is the shear 
The kinetic energy rectangular of plate is expressed as:

$K=\frac{1}{2} \int_{-\frac{h}{2}}^{\frac{h}{2}} \int_{A} \rho(z)\left(V^{P}\right)^{2} d A d z$

$\rho(z)$ is the density of FGM plate and $V^{P}$ is the velocity of force which calculate as the following:

$\overline{V^{P}}=\sqrt{\left(V_{x}^{P}\right)^{2}+\left(V_{y}^{P}\right)^{2}+\left(V_{z}^{P}\right)^{2}}$

where $V_{x}^{P}, V_{y}^{P}, V_{z}^{P}$ are the component of moving force through the $x, y, z$ axes and can be calculated from Eq. (1) as the following

$\left\{\begin{array}{l}V_{x}^{P}=\dot{u}_{x}=\dot{u}_{0}+z \dot{\psi}_{x} \\ V_{y}^{P}=\dot{u}_{y}=\dot{v}_{0}+z \dot{\psi}_{y} \\ V_{z}^{P}=\dot{u}_{z}=\dot{w}_{0}\end{array}\right.$

By replacing Eq. (10) in Eq. (9), the kinetic energy of structure is simplified as:

$\delta K=\int_{-\frac{h}{2}}^{+\frac{h}{2}} \int_{A} \rho(z)\left[\begin{array}{l}\left(\dot{u}_{0} \delta \dot{u}_{0}+\dot{u}_{0} z \delta \dot{\psi}_{x}+\delta \dot{u}_{0} z \dot{\psi}_{x}+z^{2} \dot{\psi}_{x} \delta \dot{\psi}_{x}\right) \\ +\left(\dot{v}_{0} \delta \dot{v}_{0}+\dot{v}_{0} z \delta \dot{\psi}_{y}+\delta \dot{v}_{0} z \dot{\psi}_{y}+z^{2} \dot{\psi}_{y} \delta \dot{\psi}_{y}\right)+\dot{w}_{0} \delta \dot{w}_{0}\end{array}\right] d A d z$

The external work that related to moving load $\left(P_{0}\right)$ is considered as following:

$\bar{W}=\iint_{A} P_{0} \delta(x-v t) \delta(x-e) w(x, y, t) d A$

The Hamilton's principle as the following:

$\int_{t_{1}}^{t_{2}}(\delta K-\delta U+\delta \bar{W}) d t=0$

$$
\begin{aligned}
& \left\{\begin{array}{c}
U_{0}=\frac{u_{0}}{a} \\
V_{0}=\frac{V_{0}}{b} \\
W_{0}=\frac{W_{0}}{a} \\
X=\frac{x}{g} \\
Y=\frac{y}{b} \\
\bar{P}=\frac{P_{0} h}{C_{x x}} \Rightarrow P_{0}=\frac{\bar{P} C_{x x}}{h} \\
\frac{C_{x x}}{I_{A} a^{4}} \frac{1}{T^{2}}=\frac{1}{t^{2}}
\end{array}\right.
\end{aligned}
$$

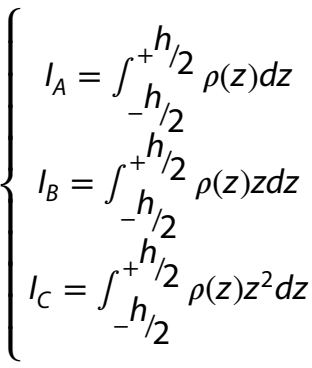

In Eq. (15), $\mathrm{U}_{0}, \mathrm{~V}_{0}, \mathrm{~W}_{0}$ are the dimensionless mid-plane displacement fields through the $x, y, z$ axes. $a, b$ and $h$ represent the length, width and thickness of the rectangular plate. $X$ and $Y$ are the dimensionless variable. Also, $t$ and $T$ are the non dimensionless and dimensionless time. Besides, $\bar{P}$ is the dimensionless force.

By substituting Eqs. (9), (12), (13) in Eq. (14) and dimensionless form of variables that is mentioned in Eqs. (15) and (16). Also, calculus of variations, the governing equations of motion are extracted as following (Eqs. 17-21):

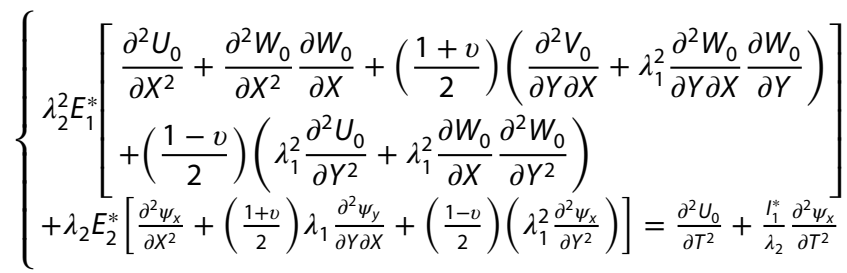

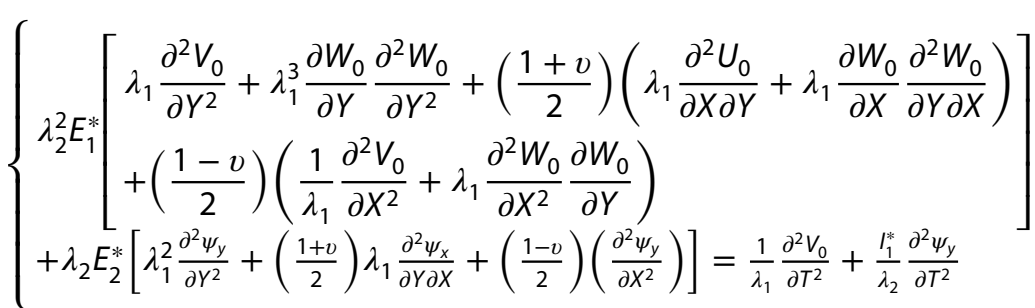




$$
\begin{aligned}
& \int \lambda_{2}^{2} E_{1}^{*}\left[\kappa\left(\frac{1-v}{2}\right)\left(\frac{\partial^{2} W_{0}}{\partial X^{2}}+\frac{\partial \psi_{x}}{\partial X}+\lambda_{1}^{2} \frac{\partial^{2} W_{0}}{\partial Y^{2}}+\lambda_{1} \frac{\partial \psi_{y}}{\partial Y}\right)\right] \\
& \left\{\begin{array}{l}
\frac{\partial^{2} U_{0}}{\partial X^{2}} \frac{\partial W_{0}}{\partial X}+\frac{\partial U_{0}}{\partial X} \frac{\partial^{2} W_{0}}{\partial X^{2}}+v \frac{\partial V_{0}}{\partial Y} \frac{\partial^{2} W_{0}}{\partial X^{2}}+\frac{3}{2}\left(\frac{\partial W_{0}}{\partial X}\right)^{2} \frac{\partial^{2} W_{0}}{\partial X^{2}} \\
+\lambda_{1}^{2}\left(\frac{1}{2}\right)\left(\frac{\partial W_{0}}{\partial Y}\right)^{2} \frac{\partial^{2} W_{0}}{\partial X^{2}}+\lambda_{1}^{2} \frac{\partial^{2} V_{0}}{\partial Y^{2}} \frac{\partial W_{0}}{\partial Y}+\lambda_{1}^{2} \frac{\partial V_{0}}{\partial Y} \frac{\partial^{2} W_{0}}{\partial Y^{2}} \\
+\lambda_{1}^{2} v \frac{\partial U_{0}}{\partial X} \frac{\partial^{2} W_{0}}{\partial Y^{2}}+\lambda_{1}^{4} \frac{3}{2}\left(\frac{\partial W_{0}}{\partial Y}\right)^{2} \frac{\partial^{2} W_{0}}{\partial Y^{2}}+\lambda_{1}^{2} \frac{1}{2}\left(\frac{\partial W_{0}}{\partial X}\right)^{2} \frac{\partial^{2} W_{0}}{\partial Y^{2}} \\
+\lambda_{1}^{2} 2 \frac{\partial W_{0}}{\partial X} \frac{\partial W_{0}}{\partial Y} \frac{\partial^{2} W_{0}}{\partial X \partial Y}+\left(\frac{1+v}{2}\right)\left(\frac{\partial^{2} V_{0}}{\partial Y \partial X} \frac{\partial W_{0}}{\partial X}+\lambda_{1}^{2} \frac{\partial^{2} U_{0}}{\partial X \partial Y} \frac{\partial W_{0}}{\partial Y}\right) \\
+\left(\frac{1-v}{2}\right)\left(\frac{\partial^{2} V_{0}}{\partial X^{2}} \frac{\partial W_{0}}{\partial Y}+\lambda_{1}^{2} \frac{\partial^{2} U_{0}}{\partial Y^{2}} \frac{\partial W_{0}}{\partial X}+2 \lambda_{1}^{2} \frac{\partial U_{0}}{\partial Y} \frac{\partial^{2} W_{0}}{\partial X \partial Y}+2 \frac{\partial V_{0}}{\partial X} \frac{\partial^{2} W_{0}}{\partial X \partial Y}\right)
\end{array}\right] \\
& \frac{\partial^{2} \psi_{x}}{\partial X^{2}} \frac{\partial W_{0}}{\partial X}+\frac{\partial \psi_{x}}{\partial X} \frac{\partial^{2} W_{0}}{\partial X^{2}}+\lambda_{1} v \frac{\partial \psi_{y}}{\partial Y} \frac{\partial^{2} W_{0}}{\partial X^{2}}+\lambda_{1}^{3} \frac{\partial^{2} \psi_{y}}{\partial Y^{2}} \frac{\partial W_{0}}{\partial Y} \\
& +\lambda_{2} E_{2}^{*}\left(+\lambda_{1}^{3} \frac{\partial \psi_{y}}{\partial Y} \frac{\partial^{2} W_{0}}{\partial Y^{2}}+v \lambda_{1}^{2} \frac{\partial \psi_{x}}{\partial X} \frac{\partial^{2} W_{0}}{\partial Y^{2}}+\left(\frac{1+v}{2}\right)\left(\lambda_{1} \frac{\partial^{2} \psi_{y}}{\partial Y \partial X} \frac{\partial W_{0}}{\partial X}+\lambda_{1}^{2} \frac{\partial^{2} \psi_{x}}{\partial Y \partial X} \frac{\partial W_{0}}{\partial Y}\right)\right. \\
& \left.+\left(\frac{1-v}{2}\right)\left(\lambda_{1} \frac{\partial^{2} \psi_{y}}{\partial X^{2}} \frac{\partial W_{0}}{\partial Y}+\lambda_{1}^{2} \frac{\partial^{2} \psi_{x}}{\partial Y^{2}} \frac{\partial W_{0}}{\partial X}+2 \lambda_{1}^{2} \frac{\partial \psi_{x}}{\partial Y} \frac{\partial^{2} W_{0}}{\partial X \partial Y}+2 \lambda_{1} \frac{\partial \psi_{y}}{\partial X} \frac{\partial^{2} W_{0}}{\partial X \partial Y}\right)\right] \\
& +\lambda_{1} \lambda_{2} \overline{P_{0}} \delta(x-v t) \delta(x-e)=\frac{\partial^{2} W_{0}}{\partial T^{2}}
\end{aligned}
$$

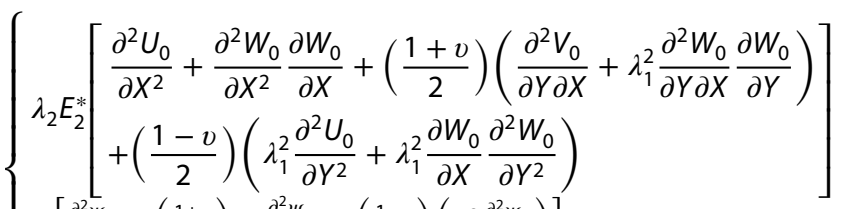$$
+\left[\frac{\partial^{2} \psi_{X}}{\partial X^{2}}+\left(\frac{1+v}{2}\right) \lambda_{1} \frac{\partial^{2} \psi_{y}}{\partial Y \partial X}+\left(\frac{1-v}{2}\right)\left(\lambda_{1}^{2} \frac{\partial^{2} \psi_{X}}{\partial Y^{2}}\right)\right]
$$$$
-\lambda_{2}^{2} E_{1}^{*}\left[\left(\frac{1-v}{2}\right) \kappa\left(\frac{\partial W_{0}}{\partial X}+\psi_{X}\right)\right]=\frac{l_{1}^{*}}{\lambda_{2}} \frac{\partial^{2} U_{0}}{\partial T^{2}}+\frac{l_{2}^{*}}{\lambda_{2}^{2}} \frac{\partial^{2} \psi_{x}}{\partial T^{2}}
$$

$$
\left\{\begin{array}{l}
\lambda_{2} E_{2}^{*}\left[\begin{array}{l}
\lambda_{1} \frac{\partial^{2} V_{0}}{\partial Y^{2}}+\lambda_{1}^{3} \frac{\partial W_{0}}{\partial Y} \frac{\partial^{2} W_{0}}{\partial Y^{2}}+\left(\frac{1+v}{2}\right)\left(\lambda_{1} \frac{\partial^{2} U_{0}}{\partial Y \partial X}+\lambda_{1} \frac{\partial^{2} W_{0}}{\partial Y \partial X} \frac{\partial W_{0}}{\partial X}\right) \\
+\left(\frac{1-v}{2}\right)\left(\frac{1}{\lambda_{1}} \frac{\partial^{2} V_{0}}{\partial X^{2}}+\lambda_{1} \frac{\partial^{2} W_{0}}{\partial X^{2}} \frac{\partial W_{0}}{\partial Y}\right)
\end{array}\right] \\
+\left[\lambda_{1}^{2} \frac{\partial^{2} \psi_{y}}{\partial Y^{2}}+\left(\frac{1+v}{2}\right) \lambda_{1} \frac{\partial^{2} \psi_{x}}{\partial \gamma X}+\left(\frac{1-v}{2}\right)\left(\frac{\partial^{2} \Psi_{y}}{\partial X^{2}}\right)\right] \\
-\lambda_{2}^{2} E_{1}^{*}\left[\left(\frac{1-v}{2}\right) \kappa\left(\lambda_{1} \frac{\partial W_{0}}{\partial Y}+\psi_{y}\right)\right]=\frac{I_{1}^{*}}{\lambda_{1} \lambda_{2}} \frac{\partial^{2} V_{0}}{\partial T^{2}}+\frac{r_{2}^{*}}{\lambda_{2}^{2}} \frac{\partial^{2} \psi_{y}}{\partial T^{2}}
\end{array}\right]
$$

$$
\begin{aligned}
& \left\{\begin{array}{l}
\lambda_{2} E_{2}^{*}\left[\begin{array}{l}
\lambda_{1} \frac{\partial^{2} V_{0}}{\partial Y^{2}}+\lambda_{1}^{3} \frac{\partial W_{0}}{\partial Y} \frac{\partial^{2} W_{0}}{\partial Y^{2}}+\left(\frac{1+v}{2}\right)\left(\lambda_{1} \frac{\partial^{2} U_{0}}{\partial Y \partial X}+\lambda_{1} \frac{\partial^{2} W_{0}}{\partial Y \partial X} \frac{\partial W_{0}}{\partial X}\right) \\
+\left(\frac{1-v}{2}\right)\left(\frac{1}{\lambda_{1}} \frac{\partial^{2} V_{0}}{\partial X^{2}}+\lambda_{1} \frac{\partial^{2} W_{0}}{\partial X^{2}} \frac{\partial W_{0}}{\partial Y}\right)
\end{array}\right] \\
+\left[\lambda_{1}^{2} \frac{\partial^{2} \psi_{Y}}{\partial Y^{2}}+\left(\frac{1+v}{2}\right) \lambda_{1} \frac{\partial^{2} \psi_{X}}{\partial Y \partial X}+\left(\frac{1-v}{2}\right)\left(\frac{\partial^{2} \psi_{y}}{\partial X^{2}}\right)\right]
\end{array}\right] \\
& -\lambda_{2}^{2} E_{1}^{*}\left[\left(\frac{1-v}{2}\right) \kappa\left(\lambda_{1} \frac{\partial W_{0}}{\partial Y}+\psi_{y}\right)\right]=\frac{l_{1}^{*}}{\lambda_{1} \lambda_{2}} \frac{\partial^{2} V_{0}}{\partial T^{2}}+\frac{l_{2}^{*}}{\lambda_{2}^{2}} \frac{\partial^{2} \psi_{y}}{\partial T^{2}}
\end{aligned}
$$

$$
\begin{aligned}
& E_{1}^{*}=\frac{\left(E_{m}+\frac{\left(E_{c}-E_{m}\right)}{n+1}\right)}{\left[\frac{1}{12} E_{m}+\left(E_{c}-E_{m}\right)\left(\frac{1}{n+3}-\frac{1}{n+2}+\frac{1}{4(n+1)}\right)\right]} \\
& E_{2}^{*}=\frac{\left(E_{c}-E_{m}\right)\left(\frac{1}{n+2}-\frac{1}{2(n+1)}\right)}{\left[\frac{1}{12} E_{m}+\left(E_{c}-E_{m}\right)\left(\frac{1}{n+3}-\frac{1}{n+2}+\frac{1}{4(n+1)}\right)\right]} \\
& \lambda_{1}=\frac{a}{b}, \lambda_{2}=\frac{a}{h} \\
& I_{1}^{*}=\frac{\left(\rho_{c}-\rho_{m}\right)\left(\frac{1}{n+2}-\frac{1}{2(n+1)}\right)}{\left(\rho_{m}+\frac{\rho_{m}}{n+1}\right)}
\end{aligned}
$$

where: 
$l_{2}^{*}=\frac{\left[\frac{1}{12} \rho_{m}+\left(\rho_{c}-\rho_{m}\right)\left(\frac{1}{n+3}-\frac{1}{n+2}+\frac{1}{4(n+1)}\right)\right]}{\left(\rho_{m}+\frac{\rho_{m}}{n+1}\right)}$

The boundary conditions are as the following (Eqs. 23 to 31 ):

$N_{x}=\left\{\begin{array}{l}A_{x x}\left(\frac{\partial u_{0}}{\partial x}+v \frac{\partial v_{0}}{\partial y}+\frac{1}{2}\left(\frac{\partial w_{0}}{\partial x}\right)^{2}+\left(\frac{v}{2}\right)\left(\frac{\partial W_{0}}{\partial y}\right)^{2}\right)+B_{x x}\left(\frac{\partial \psi_{x}}{\partial x}+v \frac{\partial \psi_{y}}{\partial y}\right)=0 \\ \rightarrow \lambda_{2}^{2} E_{1}^{*}\left(\frac{\partial U_{0}}{\partial X}+v \frac{\partial V_{0}}{\partial Y}+\frac{1}{2}\left(\frac{\partial W_{0}}{\partial X}\right)^{2}+\lambda_{1}^{2}\left(\frac{v}{2}\right)\left(\frac{\partial W_{0}}{\partial Y}\right)^{2}\right)+\lambda_{2} E_{2}^{*}\left(\frac{\partial \psi_{x}}{\partial X}+\lambda_{1} v \frac{\partial \psi_{y}}{\partial Y}\right)=0\end{array}\right.$

$N_{y}=\left\{\begin{array}{l}\frac{1}{2} A_{x x}\left(2 \frac{\partial v_{0}}{\partial y}+2 v \frac{\partial u}{\partial x}+\left(\frac{\partial w_{0}}{\partial y}\right)^{2}+v\left(\frac{\partial w_{0}}{\partial x}\right)^{2}\right)+B_{x x}\left(\frac{\partial \psi_{y}}{\partial y}+v \frac{\partial \psi_{x}}{\partial x}\right)=0 \\ \rightarrow \frac{1}{2} \lambda_{2}^{2} E_{1}^{*}\left(2 \frac{\partial V_{0}}{\partial Y}+2 v \frac{\partial U_{0}}{\partial X}+\lambda_{1}^{2}\left(\frac{\partial W_{0}}{\partial Y}\right)^{2}+v\left(\frac{\partial W_{0}}{\partial X}\right)^{2}\right)+\lambda_{2} E_{2}^{*}\left(\lambda_{1} \frac{\partial \psi_{y}}{\partial Y}+v \frac{\partial \psi_{x}}{\partial X}\right)=0\end{array}\right.$

$N_{x y}=\left\{\begin{array}{l}A_{x x}\left(\frac{1-v}{2}\right)\left(\frac{\partial u_{0}}{\partial y}+\frac{\partial v_{0}}{\partial x}+\frac{\partial w_{0}}{\partial x} \frac{\partial w_{0}}{\partial y}\right)+B_{x x}\left(\frac{1-v}{2}\right)\left(\frac{\partial \psi_{x}}{\partial y}+\frac{\partial \psi_{y}}{\partial x}\right)=0 \\ \rightarrow \lambda_{2}^{2} E_{1}^{*}\left(\lambda_{1} \frac{\partial U_{0}}{\partial Y}+\frac{1}{\lambda_{1}} \frac{\partial V_{0}}{\partial X}+\lambda_{1} \frac{\partial W_{0}}{\partial X} \frac{\partial W_{0}}{\partial Y}\right)+\lambda_{2} E_{2}^{*}\left(\lambda_{1} \frac{\partial \psi_{x}}{\partial Y}+\frac{\partial \psi_{y}}{\partial X}\right)=0\end{array}\right.$

$\boldsymbol{M}_{\boldsymbol{x}}=\left\{\begin{array}{c}\frac{1}{2} B_{x x}\left(2 \frac{\partial u_{0}}{\partial x}+2 v \frac{\partial v_{0}}{\partial y}+v\left(\frac{\partial w_{0}}{\partial y}\right)^{2}+\left(\frac{\partial w}{\partial x}\right)^{2}\right)+C_{x x}\left(\frac{\partial \psi_{x}}{\partial x}+v \frac{\partial \psi_{y}}{\partial y}\right)=0 \\ \rightarrow \frac{1}{2} \lambda_{2} E_{2}^{*}\left(2 \frac{\partial U_{0}}{\partial x}+2 v \frac{\partial V_{0}}{\partial Y}+\lambda_{1}^{2} v\left(\frac{\partial W_{0}}{\partial Y}\right)^{2}+\left(\frac{\partial W_{0}}{\partial x}\right)^{2}\right)+\left(\frac{\partial \psi_{x}}{\partial x}+\lambda_{1} v \frac{\partial \psi_{y}}{\partial Y}\right)=0\end{array}\right.$

$\boldsymbol{M}_{\boldsymbol{y}}=\left\{\begin{array}{l}\frac{1}{2} B_{x x}\left(2 v \frac{\partial u_{0}}{\partial x}+2 \frac{\partial v_{0}}{\partial y}+v\left(\frac{\partial w_{0}}{\partial x}\right)^{2}+\left(\frac{\partial w_{0}}{\partial y}\right)^{2}\right)+C_{x x}\left(v \frac{\partial \psi_{x}}{\partial x}+\frac{\partial \psi_{y}}{\partial y}\right)=0 \\ \rightarrow \frac{1}{2} \lambda_{2} E_{2}^{*}\left(2 v \frac{\partial U_{0}}{\partial X}+2 \frac{\partial V_{0}}{\partial Y}+v\left(\frac{\partial W_{0}}{\partial X}\right)^{2}+\lambda_{1}^{2}\left(\frac{\partial W_{0}}{\partial Y}\right)^{2}\right)+\left(v \frac{\partial \psi_{x}}{\partial X}+\lambda_{1} \frac{\partial \psi_{y}}{\partial Y}\right)=0\end{array}\right.$

$\boldsymbol{M}_{\boldsymbol{x y}}=\left\{\begin{array}{l}B_{x x}\left(\frac{1-v}{2}\right)\left(\frac{\partial u_{0}}{\partial y}+\frac{\partial v_{0}}{\partial x}+\frac{\partial w_{0}}{\partial x} \frac{\partial w_{0}}{\partial y}\right)+C_{x x}\left(\frac{1-v}{2}\right)\left(\frac{\partial \psi_{x}}{\partial y}+\frac{\partial \psi_{y}}{\partial x}\right)=0 \\ \rightarrow \lambda_{2} E_{2}^{*}\left(\lambda_{1} \frac{\partial U_{0}}{\partial Y}+\frac{1}{\lambda_{1}} \frac{\partial V_{0}}{\partial X}+\lambda_{1} \frac{\partial W_{0}}{\partial X} \frac{\partial W_{0}}{\partial Y}\right)+\left(\lambda_{1} \frac{\partial \psi_{x}}{\partial Y}+\frac{\partial \psi_{y}}{\partial X}\right)=0\end{array}\right.$ 
$\left\{\begin{array}{l}\kappa\left(\frac{1-v}{2}\right) A_{x x}\left(\frac{\partial w_{0}}{\partial x}+\psi_{x}\right)+A_{x x}\left(\frac{\partial u_{0}}{\partial x}+v \frac{\partial v_{0}}{\partial y}+\frac{1}{2}\left(\frac{\partial w_{0}}{\partial x}\right)^{2}+\left(\frac{v}{2}\right)\left(\frac{\partial w_{0}}{\partial y}\right)^{2}\right) \frac{\partial w_{0}}{\partial x} \\ +B_{x x}\left(\frac{\partial \psi_{x}}{\partial x}+v \frac{\partial \psi_{y}}{\partial y}\right) \frac{\partial w_{0}}{\partial x}+\frac{1-v}{2} A_{x x}\left(\frac{\partial u_{0}}{\partial y}+\frac{\partial v_{0}}{\partial x}+\frac{\partial w_{0}}{\partial x} \frac{\partial w_{0}}{\partial y}\right) \frac{\partial w_{0}}{\partial y} \\ +B_{x x}\left(\frac{1-v}{2}\right)\left(\frac{\partial \psi_{x}}{\partial y}+\frac{\partial \psi_{y}}{\partial x}\right) \frac{\partial w_{0}}{\partial y}=0 \\ \rightarrow \kappa\left(\frac{1-v}{2}\right) \lambda_{2}^{2} E_{1}^{*}\left(\frac{\partial W_{0}}{\partial X}+\psi_{x}\right) \\ +\lambda_{2}^{2} E_{1}^{*}\left(\frac{\partial U_{0}}{\partial X} \frac{\partial W_{0}}{\partial X}+v \frac{\partial V_{0}}{\partial Y} \frac{\partial W_{0}}{\partial X}+\frac{1}{2}\left(\frac{\partial W_{0}}{\partial X}\right)^{2} \frac{\partial W_{0}}{\partial X}+\lambda_{1}^{2}\left(\frac{1}{2}\right)\left(\frac{\partial W_{0}}{\partial Y}\right)^{2} \frac{\partial W_{0}}{\partial X}\right. \\ +\left(\frac{1-v}{2}\right)\left(\lambda_{1}^{2} \frac{\partial U_{0}}{\partial Y} \frac{\partial W_{0}}{\partial Y}+\frac{\partial V_{0}}{\partial X} \frac{\partial W_{0}}{\partial Y}\right)\end{array}\right)$

$$
\left\{\begin{array}{l}
\kappa\left(\frac{1-v}{2}\right) A_{x x}\left(\frac{\partial w_{0}}{\partial y}+\psi_{y}\right)+\frac{1}{2} A_{x x}\left(2 \frac{\partial v_{0}}{\partial y} \frac{\partial w_{0}}{\partial y}+2 v \frac{\partial u}{\partial x} \frac{\partial w_{0}}{\partial y}+\left(\frac{\partial w_{0}}{\partial y}\right)^{2} \frac{\partial w_{0}}{\partial y}+v\left(\frac{\partial w_{0}}{\partial x}\right)^{2} \frac{\partial w_{0}}{\partial y}\right) \\
+B_{x x}\left(\frac{\partial \psi_{y}}{\partial y} \frac{\partial w_{0}}{\partial y}+v \frac{\partial \psi_{x}}{\partial x} \frac{\partial w_{0}}{\partial y}\right)+\frac{1-v}{2} A_{x x}\left(\frac{\partial u_{0}}{\partial y} \frac{\partial w_{0}}{\partial x}+\frac{\partial v_{0}}{\partial x} \frac{\partial w_{0}}{\partial x}+\frac{\partial w_{0}}{\partial x} \frac{\partial w_{0}}{\partial y} \frac{\partial w_{0}}{\partial x}\right) \\
+B_{x x}\left(\frac{1-v}{2}\right)\left(\frac{\partial \psi_{x}}{\partial y} \frac{\partial w_{0}}{\partial x}+\frac{\partial \psi_{y}}{\partial x} \frac{\partial w_{0}}{\partial x}\right)=0 \\
\rightarrow \kappa\left(\frac{1-v}{2}\right) \lambda_{2}^{2} E_{1}^{*}\left(\lambda_{1} \frac{\partial W_{0}}{\partial Y}+\psi_{y}\right) \\
+\lambda_{2}^{2} E_{1}^{*}\left(\frac{\lambda_{1}}{1} \frac{\partial V_{0}}{\partial Y} \frac{\partial W_{0}}{\partial Y}+v \lambda_{1} \frac{\partial U}{\partial X} \frac{\partial W_{0}}{\partial Y}+\frac{1}{2} \lambda_{1}^{3}\left(\frac{\partial W_{0}}{\partial Y}\right)^{2} \frac{\partial W_{0}}{\partial Y}+\frac{1}{2} \lambda_{1}\left(\frac{\partial W_{0}}{\partial X}\right)^{2} \frac{\partial W_{0}}{\partial Y}\right. \\
+\left(\frac{1-v}{2}\right)\left(\lambda_{1} \frac{\partial U_{0}}{\partial Y} \frac{\partial W_{0}}{\partial X}+\frac{1}{\lambda_{1}} \frac{\partial V_{0}}{\partial X} \frac{\partial W_{0}}{\partial X}\right) \\
+\lambda_{2} E_{2}^{*}\left(\lambda_{1}^{2} \frac{\partial \psi_{y}}{\partial Y} \frac{\partial W_{0}}{\partial Y}+\lambda_{1} v \frac{\partial \psi_{x}}{\partial X} \frac{\partial W_{0}}{\partial Y}+\left(\frac{1-v}{2}\right)\left(\lambda_{1} \frac{\partial \psi_{x}}{\partial Y} \frac{\partial W_{0}}{\partial X}+\frac{\partial \psi_{y}}{\partial X} \frac{\partial W_{0}}{\partial X}\right)\right)=0
\end{array}\right.
$$

where

$$
\left\{\begin{array}{l}
A_{x x}=\frac{h}{1-v^{2}}\left(E_{m}+\frac{E_{c m}}{n+1}\right) \\
B_{x x}=\frac{h^{2}}{1-v^{2}} E_{c m}\left(\frac{1}{n+2}-\frac{1}{2(n+1)}\right) \\
C_{x x}=\frac{h^{3}}{1-v^{2}}\left[\frac{1}{12} E_{m}+E_{c m}\left(\frac{1}{n+3}-\frac{1}{n+2}+\frac{1}{4(n+1)}\right)\right]
\end{array}\right.
$$

\subsection{Employing GDQ method:}

Since the GDQ method is concerned, this approach discretizes the spatial derivatives of a function $f(z, t)$ as a weighted linear sum of the functional values at all nodes in the solution domain, by means of some fixed weighting coefficients. Thus, the first- and second-order derivatives of a one-dimensional function, read as follows

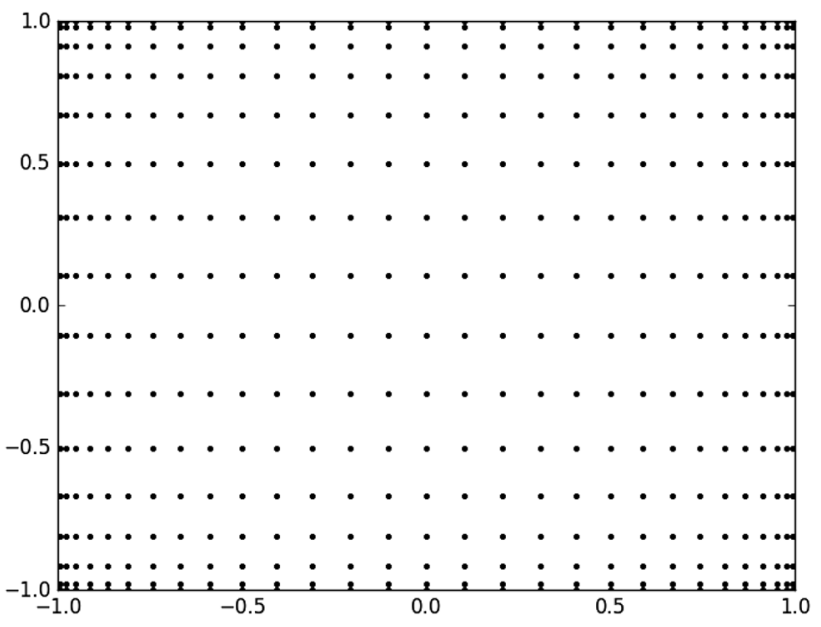

Fig. 2 Chebyshev-Gauss-Lobatto quadrature-mesh size 


$$
\begin{gathered}
\left.\frac{\partial f(z, t)}{\partial z}\right|_{z=z_{i}}=\sum_{j=1}^{N_{z}} A_{i j}^{z} f\left(z_{j}, t\right)=\sum_{j=1}^{N_{z}} A_{i j}^{z} f_{j}(t) \\
\left.\frac{\partial^{2} f(z, t)}{\partial z^{2}}\right|_{z=z_{i}}=\sum_{j=1}^{N_{z}} B_{i j}^{z} f\left(z_{j}, t\right)=\sum_{j=1}^{N_{z}} B_{i j}^{z} f_{j}(t)
\end{gathered}
$$

where $A_{i j}^{z}, B_{i j}^{z}$, are the weighted coefficients at the grid nodes of solution domain. To derive the weighting coefficients, the following relations are employed

$A_{i j}^{z}=\left\{\begin{array}{ll}\frac{M\left(z_{i}\right)}{\left(z_{i}-z_{j}\right) M\left(z_{i}\right)} \text { for } i \neq j \\ -\sum_{k=1, k \neq i}^{N_{z}} A_{i k}^{z} \text { for } i=j\end{array} \quad i, j=1,2, \ldots, N_{z^{\prime}}\right.$
$B_{i j}^{z}=\left\{\begin{array}{c}2\left[A_{i i}^{z} A_{i j}^{z}-\frac{A_{i j}^{z}}{z_{i}-z_{j}}\right] \text { for } i \neq j, \\ -\sum_{k=1, k \neq i}^{N_{z}} B_{i k}^{z} \text { for } i=j\end{array} \quad i, j=1,2, \ldots, N_{z}\right.$

being $M^{(1)}\left(z_{i}\right)=\prod_{j=1, j \neq i}^{N}\left(z_{i}-z_{j}\right)$ for $i=1,2, \ldots, N$.

To obtain more accurate results, a Chebyshev-Gauss-Lobatto quadrature-mesh size is here assumed, in line with findings by Malik and Bert [36] (see Fig. 2)

$z_{i}=\frac{h}{2}\left[1-\cos \left[\frac{(i-1) \pi}{\left(N_{z}-1\right)}\right]\right]$.

By applying GDQ method [36-38] to the boundary conditions and governing equations, we have:

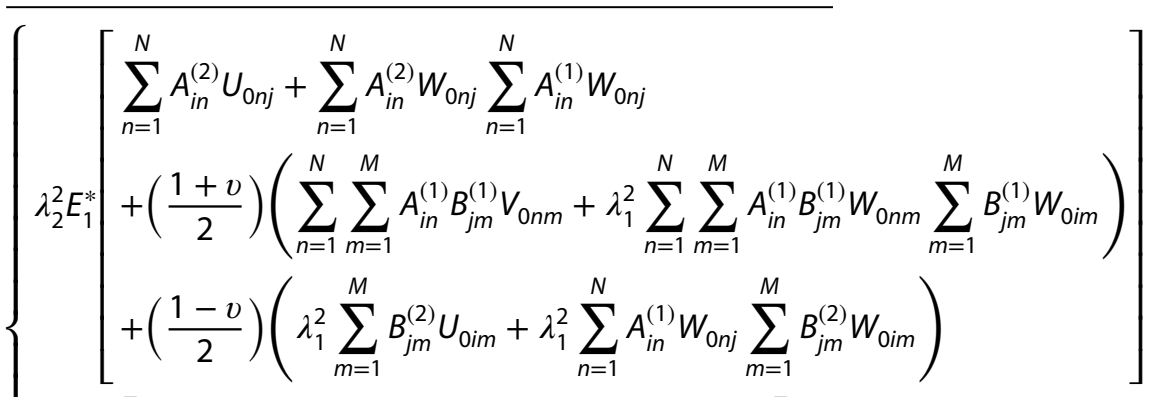

$$
\begin{aligned}
& +\lambda_{2} E_{2}^{*}\left[\begin{array}{l}
\sum_{n=1}^{N} A_{i n}^{(2)} \psi_{x n j}+\left(\frac{1+v}{2}\right) \lambda_{1} \sum_{n=1}^{N} \sum_{m=1}^{M} A_{i n}^{(1)} B_{j m}^{(1)} \psi_{y n m} \\
+\left(\frac{1-v}{2}\right)\left(\lambda_{1}^{2} \sum_{m=1}^{M} B_{j m}^{(2)} \psi_{x i m}\right)
\end{array}\right]=\frac{\partial^{2} U_{0}}{\partial T^{2}}+\frac{l_{1}^{*}}{\lambda_{2}} \frac{\partial^{2} \psi_{x}}{\partial T^{2}} \\
& \left\{\begin{array}{l}
\lambda_{2}^{2} E_{1}^{*}\left[\begin{array}{l}
\lambda_{1} \sum_{m=1}^{M} B_{j m}^{(2)} V_{0 i m}+\lambda_{1}^{3} \sum_{m=1}^{M} B_{j m}^{(1)} W_{0 i m} \sum_{m=1}^{M} B_{j m}^{(2)} W_{0 i m} \\
+\left(\frac{1+v}{2}\right)\left(\lambda_{1} \sum_{n=1}^{N} \sum_{m=1}^{M} A_{i n}^{(1)} B_{j m}^{(1)} U_{0 n m}+\lambda_{1} \sum_{n=1}^{N} A_{i n}^{(1)} W_{0 n j} \sum_{n=1}^{N} \sum_{m=1}^{M} A_{i n}^{(1)} B_{j m}^{(1)} W_{0 n m}\right) \\
+\left(\frac{1-v}{2}\right)\left(\frac{1}{\lambda_{1}} \sum_{n=1}^{N} A_{i n}^{(2)} V_{0 n j}+\lambda_{1} \sum_{n=1}^{N} A_{i n}^{(2)} W_{0 n j} \sum_{m=1}^{M} B_{j m}^{(1)} W_{0 i m}\right) \\
+\lambda_{2} E_{2}^{*}\left[\begin{array}{l}
\lambda_{1}^{2} \sum_{m=1}^{M} B_{j m}^{(2)} \psi_{y i m}+\left(\frac{1+v}{2}\right) \lambda_{1} \sum_{n=1}^{N} \sum_{m=1}^{M} A_{i n}^{(1)} B_{j m}^{(1)} \psi_{x n m} \\
+\left(\frac{1-v}{2}\right)\left(\sum_{n=1}^{N} A_{i n}^{(2)} \psi_{y n j}\right)
\end{array}\right]=\frac{1}{\lambda_{1}} \frac{\partial^{2} V_{0}}{\partial T^{2}}+\frac{l_{1}^{*}}{\lambda_{2}} \frac{\partial^{2} \psi_{y}}{\partial T^{2}}
\end{array}\right.
\end{array}\right.
\end{aligned}
$$




$$
\begin{aligned}
& \int \lambda_{2}^{2} E_{1}^{*}\left[\kappa\left(\frac{1-v}{2}\right)\left(\sum_{n=1}^{N} A_{i n}^{(2)} W_{0 n j}+\sum_{n=1}^{N} A_{i n}^{(1)} \psi_{x n j}+\lambda_{1}^{2} \sum_{m=1}^{M} B_{j m}^{(2)} W_{0 ~ i m}+\lambda_{1} \sum_{m=1}^{M} B_{j m}^{(1)} \psi_{y i m}\right)\right] \\
& {\left[\sum_{n=1}^{N} A_{i n}^{(2)} U_{0 n j} \sum_{n=1}^{N} A_{i n}^{(1)} W_{0 n j}+\sum_{n=1}^{N} A_{i n}^{(1)} U_{0 n j} \sum_{n=1}^{N} A_{i n}^{(2)} W_{0 n j}+v \sum_{m=1}^{M} B_{j m}^{(1)} V_{0 i m} \sum_{n=1}^{N} A_{i n}^{(2)} W_{0 n j}\right.} \\
& +\frac{3}{2}\left(\sum_{n=1}^{N} A_{i n}^{(1)} W_{0 n j}\right)^{2} \sum_{n=1}^{N} A_{i n}^{(2)} W_{0 n j}+\lambda_{1}^{2}\left(\frac{1}{2}\right)\left(\sum_{m=1}^{M} B_{j m}^{(1)} W_{0 i m}\right)^{2} \sum_{n=1}^{N} A_{i n}^{(2)} W_{0 n j} \\
& +\lambda_{1}^{2} \sum_{m=1}^{M} B_{j m}^{(2)} V_{0 i m} \sum_{m=1}^{M} B_{j m}^{(1)} W_{0 i m}+\lambda_{1}^{2} \sum_{m=1}^{M} B_{j m}^{(1)} V_{0 i m} \sum_{m=1}^{M} B_{j m}^{(2)} W_{0 i m} \\
& +\lambda_{1}^{2} v \sum_{n=1}^{N} A_{i n}^{(1)} U_{0 n j} \sum_{m=1}^{M} B_{j m}^{(2)} W_{0 i m}+\lambda_{1}^{4} \frac{3}{2}\left(\sum_{m=1}^{M} B_{j m}^{(1)} W_{0 i m}\right)^{2} \sum_{m=1}^{M} B_{j m}^{(2)} W_{0 i m} \\
& +\lambda_{1}^{2} \frac{1}{2}\left(\sum_{n=1}^{N} A_{i n}^{(1)} W_{0 n j}\right)^{2} \sum_{m=1}^{M} B_{j m}^{(2)} W_{0 i m}+\lambda_{1}^{2} 2 \sum_{n=1}^{N} A_{i n}^{(1)} W_{0 n j} \sum_{m=1}^{M} B_{j m}^{(1)} W_{0 i m} \sum_{n=1}^{N} \sum_{m=1}^{M} A_{i n}^{(1)} B_{j m}^{(1)} W_{0 n m} \\
& +\left(\frac{1+v}{2}\right)\left(\sum_{n=1}^{N} \sum_{m=1}^{M} A_{i n}^{(1)} B_{j m}^{(1)} V_{0 n m} \sum_{n=1}^{N} A_{i n}^{(1)} W_{0 n j}+\lambda_{1}^{2} \sum_{n=1}^{N} \sum_{m=1}^{M} A_{i n}^{(1)} B_{j m}^{(1)} U_{0 n m} \sum_{m=1}^{M} B_{j m}^{(1)} W_{0 ~ i m}\right) \\
& +\left(\frac{1-v}{2}\right)\left(\sum_{n=1}^{N} A_{i n}^{(2)} V_{0 n j} \sum_{m=1}^{M} B_{j m}^{(1)} W_{0 ~ i m}+\lambda_{1}^{2} \sum_{m=1}^{M} B_{j m}^{(2)} U_{0 i m} \sum_{n=1}^{N} A_{i n}^{(1)} W_{0 n j}\right. \\
& \left.\left(+2 \lambda_{1}^{2} \sum_{m=1}^{M} B_{j m}^{(1)} U_{0} i m \sum_{n=1}^{N} \sum_{m=1}^{M} A_{i n}^{(1)} B_{j m}^{(1)} W_{0 n m}+2 \sum_{n=1}^{N} A_{i n}^{(1)} V_{0 n j} \sum_{n=1}^{N} \sum_{m=1}^{M} A_{i n}^{(1)} B_{j m}^{(1)} W_{0 n m}\right)\right] \\
& {\left[\sum_{n=1}^{N} A_{i n}^{(2)} \psi_{x n j} \sum_{n=1}^{N} A_{i n}^{(1)} W_{0 n j}+\sum_{n=1}^{N} A_{i n}^{(1)} \psi_{x n j} \sum_{n=1}^{N} A_{i n}^{(2)} W_{0 n j}+\right.} \\
& \lambda_{1} v \sum_{m=1}^{M} B_{j m}^{(1)} \psi_{y} \sum_{i m}^{N} A_{i n}^{(2)} W_{0 n j} \\
& +\lambda_{1}^{3} \sum_{m=1}^{M} B_{j m}^{(2)} \psi_{y} i m \sum_{m=1}^{M} B_{j m}^{(1)} W_{0 ~ i m}+\lambda_{1}^{3} \sum_{m=1}^{M} B_{j m}^{(1)} \psi_{y} i m \sum_{m=1}^{M} B_{j m}^{(2)} W_{0 i m} \\
& +v \lambda_{1}^{2} \sum_{n=1}^{N} A_{i n}^{(1)} \psi_{x n j} \sum_{m=1}^{M} B_{j m}^{(2)} W_{0 ~ i m} \\
& +\lambda_{2} E_{2}^{*}\left(\frac{1+v}{2}\right)\left(\begin{array}{l}
\lambda_{1} \sum_{n=1}^{N} \sum_{m=1}^{M} A_{i n}^{(1)} B_{j m}^{(1)} \psi_{y} n m \sum_{n=1}^{N} A_{i n}^{(1)} W_{0 n j} \\
+\lambda_{1}^{2} \sum_{n=1}^{N} \sum_{m=1}^{M} A_{i n}^{(1)} B_{j m}^{(1)} \psi_{x n m} \sum_{m=1}^{M} B_{j m}^{(1)} W_{0 ~ i m}
\end{array}\right) \\
& \left.+\left(\frac{1-v}{2}\right)\left(\begin{array}{l}
\lambda_{1} \sum_{n=1}^{N} A_{i n}^{(2)} \psi_{y} \sum_{m=1}^{M} B_{j m}^{(1)} W_{0 ~ i m}+\lambda_{1}^{2} \sum_{m=1}^{M} B_{j m}^{(2)} \psi_{x i m} \sum_{n=1}^{N} A_{i n}^{(1)} W_{0 n j} \\
+2 \lambda_{1}^{2} \sum_{m=1}^{M} B_{j m}^{(1)} \psi_{x i m} \sum_{n=1}^{N} \sum_{m=1}^{M} A_{i n}^{(1)} B_{j m}^{(1)} W_{0 n m}+ \\
2 \lambda_{1} \sum_{n=1}^{N} A_{i n}^{(1)} \psi_{y} \sum_{n=1}^{N} \sum_{m=1}^{M} A_{i n}^{(1)} B_{j m}^{(1)} W_{0 n m}
\end{array}\right)\right] \\
& +\lambda_{1} \lambda_{2}^{2} \bar{P}_{i j} \Delta x_{i} \Delta y_{j}=\frac{\partial^{2} W_{0}}{\partial T^{2}}
\end{aligned}
$$


SN Applied Sciences (2021) 3:847

| https://doi.org/10.1007/s42452-021-04825-9

Research Article

$$
\begin{aligned}
& \left\{\begin{array}{l}
\lambda_{2} E_{2}^{*}\left[\begin{array}{l}
\sum_{n=1}^{N} A_{i n}^{(2)} U_{0 n j}+\sum_{n=1}^{N} A_{i n}^{(2)} W_{0 n j} \sum_{n=1}^{N} A_{i n}^{(1)} W_{0 n j} \\
+\left(\frac{1+v}{2}\right)\left(\sum_{n=1}^{N} \sum_{m=1}^{M} A_{i n}^{(1)} B_{j m}^{(1)} V_{0 n m}+\lambda_{1}^{2} \sum_{n=1}^{N} \sum_{m=1}^{M} A_{i n}^{(1)} B_{j m}^{(1)} W_{0 n m} \sum_{m=1}^{M} B_{j m}^{(1)} W_{0 i m}\right) \\
+\left(\frac{1-v}{2}\right)\left(\lambda_{1}^{2} \sum_{m=1}^{M} B_{j m}^{(2)} U_{0 i m}+\lambda_{2}^{2} \sum_{n=1}^{N} A_{i n}^{(1)} W_{0 n j} \sum_{m=1}^{M} B_{j m}^{(2)} W_{0 i m}\right)
\end{array}\right. \\
+\left[\begin{array}{l}
\sum_{n=1}^{N} A_{i n}^{(2)} \psi_{x n j}+\left(\frac{1+v}{2}\right) \lambda_{1} \sum_{n=1}^{N} \sum_{m=1}^{M} A_{i n}^{(1)} B_{j m}^{(1)} \psi_{y n m}+\left(\frac{1-v}{2}\right)\left(\lambda_{1}^{2} \sum_{m=1}^{M} B_{j m}^{(2)} \psi_{x i m}\right)
\end{array}\right] \\
-\left[\left(\frac{1-v}{2}\right) \lambda_{2}^{2} E_{1}^{*} K\left(\sum_{n=1}^{N} A_{i n}^{(1)} W_{0 n j}+\psi_{x}\right)\right]=\frac{l_{1}^{*}}{\lambda_{2}} \frac{\partial^{2} U_{0}}{\partial T^{2}}+\frac{l_{2}^{*}}{\lambda_{2}^{2}} \frac{\partial^{2} \Psi_{x}}{\partial T^{2}}
\end{array}\right] \\
& \left\{\begin{array}{c}
\lambda_{2} E_{2}^{*}\left[\begin{array}{l}
\lambda_{1} \sum_{m=1}^{M} B_{j m}^{(2)} V_{\text {oim }}+\lambda_{1}^{3} \sum_{m=1}^{M} B_{j m}^{(1)} W_{\text {oim }} \sum_{m=1}^{M} B_{j m}^{(2)} W_{\text {oim }} \\
+\left(\frac{1+v}{2}\right)\left(\lambda_{1} \sum_{n=1}^{N} \sum_{m=1}^{M} A_{i n}^{(1)} B_{j m}^{(1)} U_{0 n m}+\lambda_{1} \sum_{n=1}^{N} \sum_{m=1}^{M} A_{i n}^{(1)} B_{j m}^{(1)} W_{\text {onm }} \sum_{n=1}^{N} A_{i n}^{(1)} W_{\text {onj }}\right) \\
+\left(\frac{1-v}{2}\right)\left(\frac{1}{\lambda_{1}} \sum_{n=1}^{N} A_{i n}^{(2)} V_{0 n j}+\lambda_{1} \sum_{n=1}^{N} A_{i n}^{(2)} W_{\text {onj }} \sum_{m=1}^{M} B_{j m}^{(1)} W_{\text {oim }}\right)
\end{array}\right] \\
+\left[\lambda_{1}^{2} \sum_{m=1}^{M} B_{j m}^{(2)} \psi_{\text {yim }}+\left(\frac{1+v}{2}\right) \lambda_{1} \sum_{n=1}^{N} \sum_{m=1}^{M} A_{i n}^{(1)} B_{j m}^{(1)} \Psi_{x n m}+\left(\frac{1-v}{2}\right)\left(\sum_{n=1}^{N} A_{i n}^{(2)} \psi_{\text {ynj }}\right)\right] \\
-\lambda_{2}^{2} E_{1}^{*}\left[\left(\frac{1-v}{2}\right) \kappa\left(\lambda_{1} \sum_{m=1}^{M} B_{j m}^{(1)} W_{\text {oim }}+\psi_{y}\right)\right]=\frac{l_{1}^{*}}{\lambda_{1} \lambda_{2}} \frac{\partial^{2} V_{0}}{\partial T^{2}}+\frac{l_{2}^{*}}{\lambda_{2}^{2}} \frac{\partial^{2} \psi_{y}}{\partial T^{2}}
\end{array}\right.
\end{aligned}
$$

(40)

Also, the boundary condition can be discretized by using GDQM as following:

$$
\begin{aligned}
& N_{x}=\left\{\begin{array}{l}
\lambda_{2}^{2} E_{1}^{*}\left(\sum_{n=1}^{N} A_{i n}^{(1)} U_{0 n j}+v \sum_{m=1}^{M} B_{j m}^{(1)} V_{0 i m}+\frac{1}{2}\left(\sum_{n=1}^{N} A_{i n}^{(1)} W_{0 n j}\right)^{2}+\lambda_{1}^{2}\left(\frac{v}{2}\right)\left(\sum_{m=1}^{M} B_{j m}^{(1)} W_{0 i m}\right)^{2}\right. \\
+\lambda_{2} E_{2}^{*}\left(\sum_{n=1}^{N} A_{i n}^{(1)} \psi_{x n j}+\lambda_{1} v \sum_{m=1}^{M} B_{j m}^{(1)} \psi_{y i m}\right)=0
\end{array}\right. \\
& \boldsymbol{N}_{\mathbf{y}}=\left\{\begin{array}{l}
\frac{1}{2} \lambda_{2}^{2} E_{1}^{*}\left(2 \sum_{m=1}^{M} B_{j m}^{(1)} V_{0 i m}+2 v \sum_{n=1}^{N} A_{i n}^{(1)} U_{0 n j}+\lambda_{1}^{2}\left(\sum_{m=1}^{M} B_{j m}^{(1)} W_{0 i m}\right)^{2}+v\left(\sum_{n=1}^{N} A_{i n}^{(1)} W_{0 n j}\right)^{2}\right. \\
+\lambda_{2} E_{2}^{*}\left(\lambda_{1} \sum_{m=1}^{M} B_{j m}^{(1)} \psi_{y i m}+v \sum_{n=1}^{N} A_{i n}^{(1)} \psi_{x n j}\right)=0
\end{array}\right.
\end{aligned}
$$

SN Applied Sciences

A SPRINGER NATURE journal 
$\boldsymbol{N}_{\boldsymbol{x y}}=\left\{\begin{array}{l}\lambda_{2}^{2} E_{1}^{*}\left(\lambda_{1} \sum_{m=1}^{M} B_{j m}^{(1)} U_{0 i m}+\frac{1}{\lambda_{1}} \sum_{n=1}^{N} A_{i n}^{(1)} V_{\text {onj }}+\lambda_{1} \sum_{n=1}^{N} A_{i n}^{(1)} W_{0 n j} \sum_{m=1}^{M} B_{j m}^{(1)} W_{0 i m}\right) \\ +\lambda_{2} E_{2}^{*}\left(\lambda_{1} \sum_{m=1}^{M} B_{j m}^{(1)} \psi_{x i m}+\sum_{n=1}^{N} A_{i n}^{(1)} \psi_{\text {ynj }}\right)=0\end{array}\right.$
$\boldsymbol{M}_{\mathbf{x}}=\left\{\begin{array}{l}\frac{1}{2} \lambda_{2} E_{2}^{*}\left(2 \sum_{n=1}^{N} A_{i n}^{(1)} U_{0 n j}+2 v \sum_{m=1}^{M} B_{j m}^{(1)} V_{0 i m}+\lambda_{1}^{2} v\left(\sum_{m=1}^{M} B_{j m}^{(1)} W_{0 i m}\right)^{2}+\left(\sum_{n=1}^{N} A_{i n}^{(1)} W_{0 n j}\right)^{2}\right. \\ +\left(\sum_{n=1}^{N} A_{i n}^{(1)} \psi_{x n j}+\lambda_{1} v \sum_{m=1}^{M} B_{j m}^{(1)} \psi_{y i m}\right)=0\end{array}\right.$

$\boldsymbol{M}_{\mathbf{y}}=\left\{\begin{array}{l}\frac{1}{2} \lambda_{2} E_{2}^{*}\left(2 v \sum_{n=1}^{N} A_{i n}^{(1)} U_{0 n j}+2 \sum_{m=1}^{M} B_{j m}^{(1)} V_{0 i m}+v\left(\sum_{n=1}^{N} A_{i n}^{(1)} W_{0 n j}\right)^{2}+\lambda_{1}^{2}\left(\sum_{m=1}^{M} B_{j m}^{(1)} W_{0 i m}\right)^{2}\right) \\ +\left(v \sum_{n=1}^{N} A_{i n}^{(1)} \psi_{x n j}+\lambda_{1} \sum_{m=1}^{M} B_{j m}^{(1)} \psi_{\text {yim }}\right)=0\end{array}\right.$

$\boldsymbol{M}_{\mathbf{x y}}=\left\{\begin{array}{l}\lambda_{2} E_{2}^{*}\left(\lambda_{1} \sum_{m=1}^{M} B_{j m}^{(1)} U_{0 i m}+\frac{1}{\lambda_{1}} \sum_{n=1}^{N} A_{i n}^{(1)} V_{0 n j}+\lambda_{1} \sum_{n=1}^{N} A_{i n}^{(1)} W_{0 n j} \sum_{m=1}^{M} B_{j m}^{(1)} W_{0 i m}\right) \\ +\left(\lambda_{1} \sum_{m=1}^{M} B_{j m}^{(1)} \psi_{x i m}+\sum_{n=1}^{N} A_{i n}^{(1)} \psi_{y n j}\right)=0\end{array}\right.$

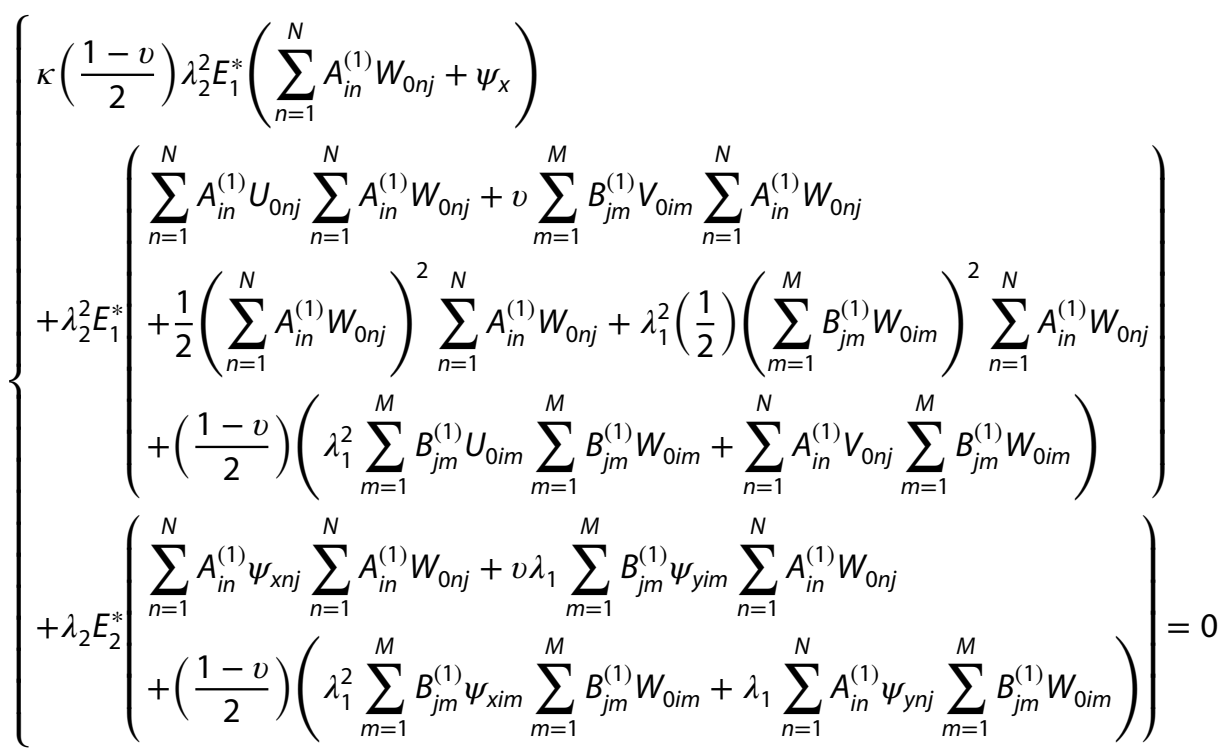




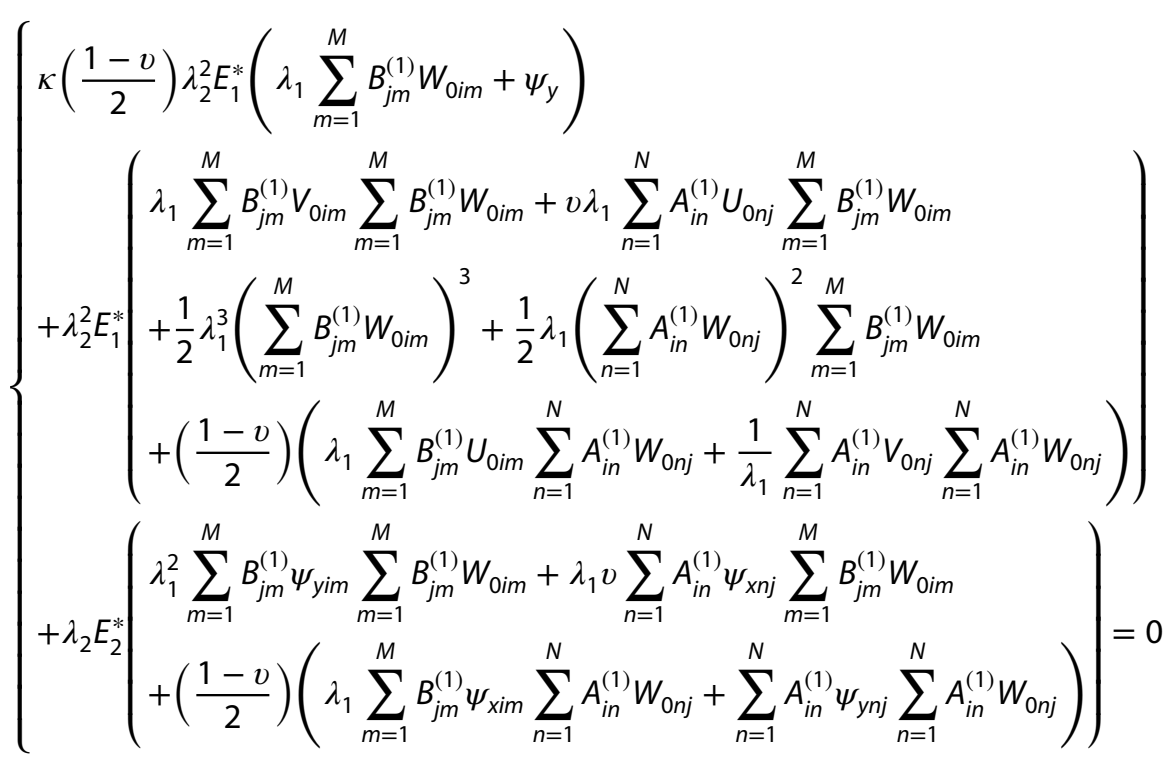

The different boundary conditions that are considered in this article can be expressed as:

a) For simply supported condition:

$\left\{\begin{array}{l}X=0,1 \psi_{y}=U_{0}=V_{0}=W_{0}=M_{x}=0 \\ Y=0,1 \psi_{x}=U_{0}=V_{0}=W_{0}=M_{y}=0\end{array}\right.$

b) For clamed boundary condition:

$\begin{cases}X=0,1 & \psi_{x}=\psi_{y}=U_{0}=V_{0}=W_{0}=0 \\ Y=0,1 & \psi_{X}=\psi_{y}=U_{0}=V_{0}=W_{0}=0\end{cases}$

c) Edges for free boundary condition

$$
\begin{cases}X=0,1 & Q_{x}=N_{x}=N_{y}=M_{x}=M_{x y}=0 \\ Y=0,1 & Q_{y}=N_{x}=N_{y}=M_{y}=M_{x y}=0\end{cases}
$$

The governing equations for free vibration analysis can be obtained by ignoring the force term in Eqs. ((36) to (40)) as the following form:

$\left[k_{b b}\right]\left[d_{b}\right]+\left[k_{b d}\right]\left[d_{d}\right]=0$

$\left[k_{d b}\right]\left[d_{b}\right]+\left[k_{d d}\right]\left[d_{b}\right]=\omega[I]\left[d_{b}\right]$

where the stiffness quantities $\mathbf{k}_{b b}, \mathbf{k}_{d b}, \mathbf{k}_{b d}, \mathbf{k}_{d d}$ refer to the boundary $b$ and domain $d$ weighting coefficients of the plate, respectively, $\left[\mathbf{d}_{b} \mathbf{d}_{d}\right]^{T}$ is the displacement vector, [I] refers to the identify matrix, and $\omega$ is the natural frequencies.

At the same time, from Eq. (52), the boundary weight coefficients can be replaced by the domain weight coefficients as follows $\mathbf{d}_{b}=\mathbf{k}_{b b}^{-1} \mathbf{k}_{b d} \mathbf{d}_{d}$

Finally, by replacing Eqs. (53), (54), the dimensionless natural frequencies can be calculated as the following:

$\omega_{i}=$ eigenvalue $\left(-\left[k_{d b}\right]\left(\left[k_{b b}\right]^{-1}\left[k_{b d}\right]\right)+\left[k_{d d}\right]\right)$

For nonlinear bending, the governing equation may be obtained in GDQ matrix form:

$\left[K_{L}+K_{N L}\right]\left[d_{0}\right]+[M]\left[\ddot{d}_{0}\right]=[F]$

For solving free vibration, it is sufficient that Eq. (52) be equal zero. Also for transient vibration, the Newmark procedure should be applied [39-41].

\section{Numerical results and discussions}

\subsection{Verification of Free vibration}

In this section, the linear and nonlinear natural frequencies of rectangular plate are obtained and compared with two various references that one of which is based on linear analysis and the other one is based on nonlinear analysis. For verification and numerical results section, the naming of boundary condition starts from edge $(x=0)$, edge $(y=b)$, edge $(x=a)$, edge $(y=0)$, respectively. In other words, SFCS represents that edge $(x=0)$ is simple, edge $(y=b)$ is free, edge $(x=a)$ is clamp and edge $(y=0)$ is simple. 


\section{Example1: Verification of linear free vibration}

For this target, the properties and details are considered as those of Ref [42] $(a / h=100)$. The first eight linear dimensionless natural frequencies of a homogenous rectangular plate are obtained. The comparison between present results and Ref [42] for different length ratios $(a / b)$ and edge boundary conditions are shown in Tables 1 and 2 . As can be seen, there is a good agreement between them.

The natural frequencies are dimensionless as the following relation:

$\Omega=\omega a^{2} \sqrt{\frac{\rho h}{D}}$

\section{Example2: verification of nonlinear free vibration}

For this goal, the properties and dimensions are the same with Ref [43]. Comparing the nonlinear frequency ratio $\left(\frac{\omega_{n}}{n}\right)$ of isotropic homogenous square plate with those of Ref [43] for various boundary conditions are shown in Table 3 and excellent agreement is found between them. It is obvious that the maximum and minimum nonlinear frequency ratio is related to SSSS and CCCC boundary conditions.

\subsection{Dynamic analysis}

\subsubsection{Verification}

Example 1: For validation of dynamic results with homogeneous plate of Ref [44], power law exponent (n) should be equal to 0 . The nonlinear transient deflection of centerpoint of isotropic homogenous plate excited by a moving force are depicted in Figs. 3 and 4 for SSSS and CCCC edge boundary conditions, respectively. Besides, there is a good correspondence between the result of present work and those of Ref [44].

Example 2: For validation of linear and nonlinear dynamic results with FGM simply supported plate of Ref [3], it is sufficient to consider the material property, geometry, velocity of the moving load is as the following:

Geometry: $\mathrm{a}=1, \mathrm{~b}=0.5, \mathrm{~h}=0.01$.

Material property:

$\mathrm{E}_{\mathrm{C}}=200 \mathrm{GPa}, v=0.3, \rho=5700$.

$\mathrm{E}_{\mathrm{m}}=70 \mathrm{Gpa}, v=0.3, \rho=2702$.

$\mathrm{P}=1 \mathrm{Kn}, \mathrm{V}=20 \mathrm{~m} / \mathrm{s}$.

The comparison between present results and Ref for linear and nonlinear analysis [3] is depicted in Fig. 5, and shows a good agreement.

\subsubsection{Numerical results}

The convergence study of present model for natural frequencies in non-dimensional form for various edge conditions is presented in Fig. 6. As it is obvious, the results for 19 grid points are convergent. The geometry and mechanical properties of FGM plate are assumed as following:

$(\mathrm{a} / \mathrm{b}=1, \mathrm{~h} / \mathrm{a}=0.1), E_{c}=200 \mathrm{GPa}, E_{m}=70 \mathrm{GPa}$,

$\rho_{m}=2702, \rho_{c}=5700, v=0.3$

The influence of velocity of moving force for different boundary conditions and $\mathrm{n}=2$ on transient center-point transverse displacement of plate are depicted in Figs. 7, 8, 9,10 and 11 , respectively. As it is obvious, the amplitude of nonlinear deflection is smaller than the linear one, and by increasing the velocity of moving force, the deflection significantly increases. Also, the CCCC and SSSS boundary conditions have the minimum and maximum amplitudes of transient deflection. Also, when the moving load has low speed, the deflection of middle point of the plate reaches the initial state (a flat plate), while at higher speeds of the moving load, the deflection of middle point is not zero because of the vibrations created during this loading. The influence of material gradient index on linear and nonlinear transient deflection are shown in Figs. 12 and 13 for SSSS and CCCC boundary conditions. By enhancing the material gradient index, the transient deflection significantly decreases. This is due to the fact that as the power law index increases, the volume fraction of ceramic constituent also increases, and therefore, stiffness of plate enhances. The effect of magnitude of moving force for two various velocities are shown in Figs. 14 and 15. It is seen that as the magnitude of force increases, the transient deflection grows. The effect of length ratio on the time history of deflection of center-point of plate for SSSS condition ( $V=1 \mathrm{~m} / \mathrm{s}, \mathrm{V}=4 \mathrm{~m} / \mathrm{s})$ are shown in Figs. 16 and 17. As can be seen, by raising the aspect ratio, the transient central deflection reduces.

The linear and nonlinear investigation on transient distribution of $\sigma_{x x}$ of the top and bottom surfaces of plate for various material gradient index, different boundary conditions and moving load are shown in Figs. 18, 19, 20, 21. As can be seen, the amount of linear stresses are larger than nonlinear ones due to the linear transient deflection is larger than nonlinear one. Also, it can be observed that the differences between linear and nonlinear amounts for the bottom of plate is larger than its top. The most difference between linear and nonlinear amount of $\sigma_{x x}$ is approximately (52\%). Besides, by increasing the material gradient index, the amount of stresses considerably decrease. Furthermore, among all the edge conditions, 
Table 1 The first eight linear dimensionless natural frequencies of a homogenous rectangular plate for various (a/b) compared with Ref [42] for SSSF boundary conditions

\begin{tabular}{|c|c|c|c|c|c|c|c|c|c|}
\hline \multirow[b]{2}{*}{$a / b$} & \multicolumn{9}{|l|}{ Mode number } \\
\hline & & 1 & 2 & 3 & 4 & 5 & 6 & 7 & 8 \\
\hline \multirow[t]{2}{*}{0.4} & Present & 9.9317 & 12.7256 & 18.1572 & 26.1857 & 36.7318 & 37.0603 & 39.7626 & 44.9859 \\
\hline & Hashemi [42] & 9.9310 & 12.7247 & 18.1563 & 26.1848 & 36.7312 & 37.0585 & 39.7612 & 44.9842 \\
\hline \multirow[t]{2}{*}{0.5} & Present & 10.0933 & 14.3277 & 22.5573 & 34.7432 & 37.1866 & 41.3224 & 49.2895 & 50.6218 \\
\hline & Hashemi[42] & 10.0929 & 14.3272 & 22.5568 & 34.7428 & 37.1858 & 41.3217 & 49.2886 & 50.6215 \\
\hline \multirow[t]{2}{*}{1} & Present & 11.3811 & 26.1911 & 38.361 & 53.3852 & 55.762 & 78.649 & 81.3691 & 92.5862 \\
\hline & Hashemi[42] & 11.3810 & 26.1910 & 38.3610 & 53.3852 & 55.7620 & 78.6490 & 81.3690 & 92.5861 \\
\hline \multirow[t]{2}{*}{2} & Present & 15.4054 & 42.887 & 66.372 & 82.719 & 92.9718 & 130.3462 & 131.5689 & 162.3547 \\
\hline & Hashemi[42] & 15.4054 & 42.8870 & 66.3720 & 82.7190 & 92.9718 & 130.3462 & 131.5689 & 162.3547 \\
\hline \multirow[t]{2}{*}{2.5} & Present & 17.7395 & 45.9146 & 85.65 & 93.2356 & 118.5437 & 134.1717 & 154.6262 & 188.621 \\
\hline & Hashemi[42] & 17.7395 & 45.9146 & 85.6500 & 93.2356 & 118.5437 & 134.1717 & 154.6262 & 188.6209 \\
\hline
\end{tabular}

Table 2 The first eight linear dimensionless natural frequencies of a homogenous rectangular plate for various (a/b) compared with Ref [42] for SSSS boundary condition

\begin{tabular}{|c|c|c|c|c|c|c|c|c|c|}
\hline \multirow[b]{2}{*}{$a / b$} & \multicolumn{9}{|l|}{ Mode number } \\
\hline & & 1 & 2 & 3 & 4 & 5 & 6 & 7 & 8 \\
\hline \multirow[t]{2}{*}{0.4} & Present & 11.2226 & 15.7408 & 23.1193 & 33.1515 & 38.3928 & 42.5226 & 45.5846 & 49.2869 \\
\hline & Hashemi [42] & 11.226 & 15.7408 & 23.1193 & 33.1515 & 38.3928 & 42.5226 & 45.5845 & 49.2869 \\
\hline \multirow[t]{2}{*}{0.5} & Present & 12.0752 & 19.084 & 30.4080 & 39.1713 & 45.5845 & 45.5845 & 55.9920 & 64.0824 \\
\hline & Hashemi [42] & 12.0752 & 19.0840 & 30.4080 & 39.1713 & 45.5845 & 45.5845 & 55.9920 & 64.0823 \\
\hline \multirow[t]{2}{*}{1} & Present & 19.084 & 45.5845 & 45.5845 & 70.0219 & 85.3654 & 85.3654 & 107.1775 & 107.1775 \\
\hline & Hashemi [42] & 19.0840 & 45.5845 & 45.5845 & 70.0219 & 85.3654 & 85.3654 & 107.1775 & 107.1775 \\
\hline \multirow[t]{2}{*}{2} & Present & 45.5845 & 70.0219 & 107.1775 & 134.3586 & 153.539 & 153.539 & 183.5877 & 206.1567 \\
\hline & Hashemi [42] & 45.5845 & 70.0219 & 107.1775 & 134.3586 & 153.5390 & 153.5390 & 183.5877 & 206.1567 \\
\hline \multirow[t]{2}{*}{2.5} & Present & 64.0823 & 87.2358 & 122.7104 & 167.3362 & 189.3433 & 206.1567 & 218.3524 & 232.8175 \\
\hline & Hashemi [42] & 64.0824 & 87.2358 & 122.7104 & 167.3362 & 189.3433 & 206.1567 & 218.3524 & 232.8175 \\
\hline
\end{tabular}

Table 3 Nonlinear natural frequency ratio of square plate compared with Ref [43] for different boundary conditions

\begin{tabular}{lllllll}
\hline \multicolumn{2}{l}{ Nonlinear frequency ratio } & \multicolumn{5}{l}{ Wmax/h (different amplitude ratio) } \\
\cline { 3 - 7 } & & 0.2 & 0.4 & 0.6 & 0.8 & 1 \\
\hline SSSS & Present & 1.0261 & 1.1011 & 1.2166 & 1.3631 & 1.5325 \\
& Rao[43] & 1.0259 & 1.1002 & 1.2140 & 1.3624 & 1.5314 \\
CCCC & Present & 1.0096 & 1.0379 & 1.0833 & 1.1437 & 1.2166 \\
& Rao[43] & 1.0095 & 1.0375 & 1.0825 & 1.1424 & 1.2149 \\
CSCS & Present & 1.0168 & 1.0658 & 1.1431 & 1.2438 & 1.3617 \\
& Rao[43] & 1.0128 & 1.0703 & 1.1498 & 1.2499 & 1.3650 \\
SSSC & Present & 1.0201 & 1.0781 & 1.1691 & 1.2859 & 1.4216 \\
& Rao[43] & 1.0210 & 1.0811 & 1.1735 & 1.2908 & 1.4264 \\
CCCS & Present & 1.0121 & 1.0479 & 1.1049 & 1.1804 & 1.2694 \\
& Rao[43] & 1.0125 & 1.0490 & 1.1063 & 1.1810 & 1.2694 \\
CCSS & Present & 1.0133 & 1.0524 & 1.1148 & 1.1971 & 1.2953 \\
& Rao[43] & 1.0132 & 1.0521 & 1.1141 & 1.1958 & 1.2938 \\
\hline
\end{tabular}

the highest and lowest values of stresses belong to SSSS of stresses significantly increase. The linear and nonlinear stresses of the top and bottom of plate can be also extended for $\sigma_{y y}$ that are depicted in Figs. 22, 23, 24, 25

that by increasing the velocity of moving load the values 
Fig. 3 Nonlinear transient deflection of plate compared with Ref [44] for SSSS boundary condition
Fig. 4 Nonlinear transient deflection of plate compared with Ref [44] for CCCC boundary condition
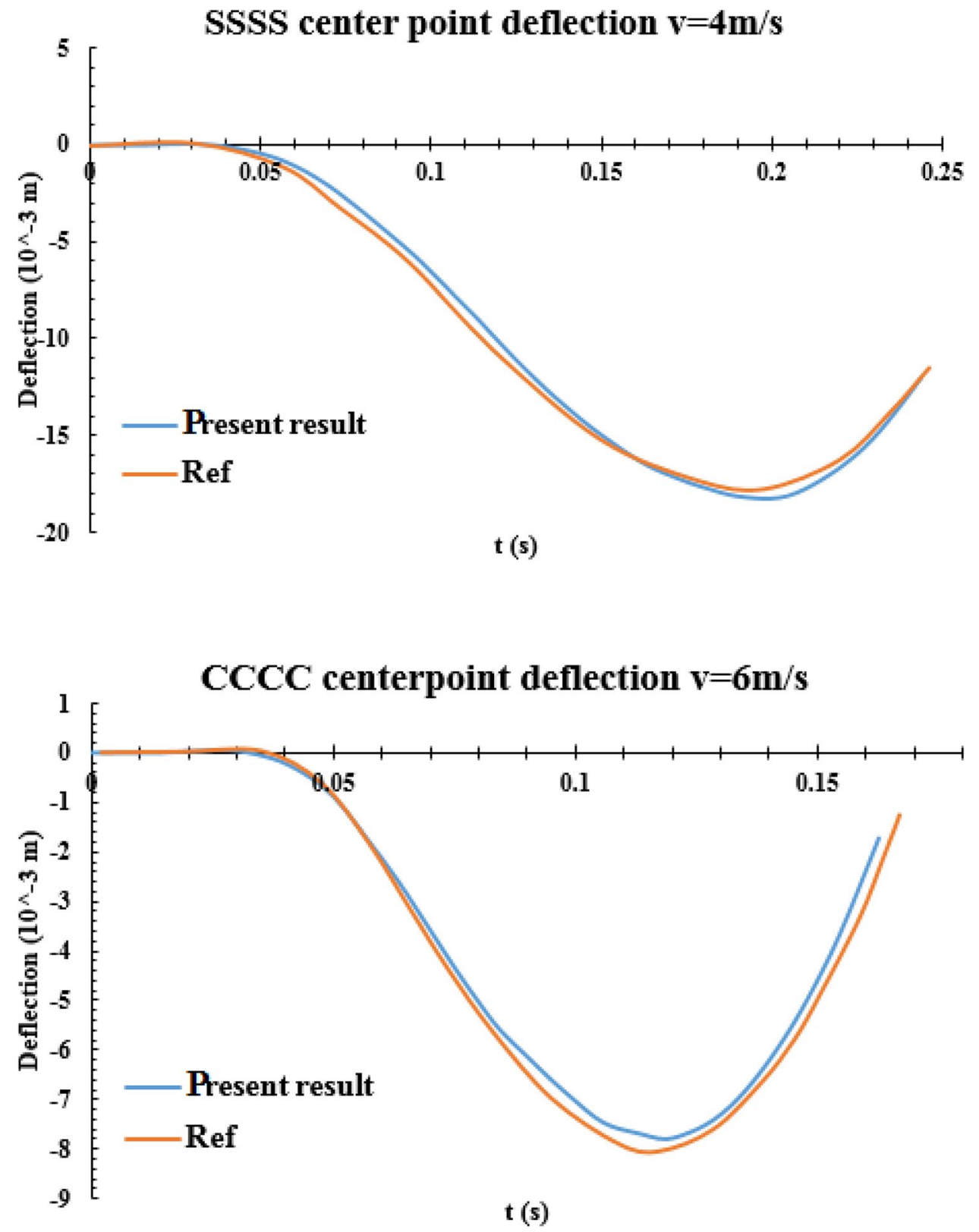
Fig. 5 Linear and nonlinear transient deflection of FGM simply supported plate compared with Ref [3]

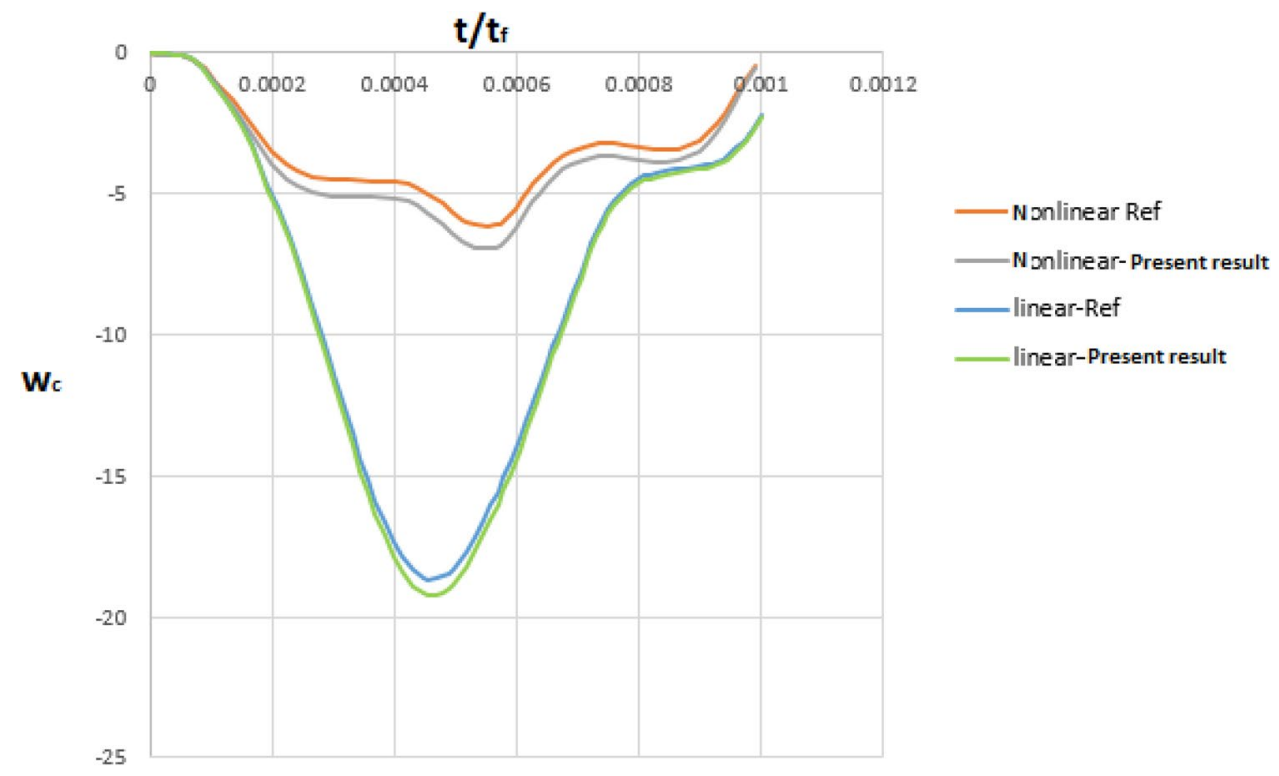

for different types of edge boundary conditions, material gradient index and velocity of moving force.

\section{Conclusion}

In this article, nonlinear dynamic bending of FGM moderately thick plates excited by a moving force using Hamilton's principle, FSDT theory and Von Karman geometric nonlinearity assumptions and by employing GDQM method for different types of edge boundary conditions is investigated. The effect of material gradient index, mass and velocity of moving load and length ratio on linear and nonlinear transient deflection and stresses have been surveyed. Also, by excluding nonlinear terms of strain and displacements relations, linear and nonlinear dynamic characteristics of plate are compared.

Some of remarkable results and novelties are as following:
- Performing linear and nonlinear analysis in conjunction with GDQM based on FSDT for solution of dynamics of plates subjected to a moving load.

- The minimum deflection and stresses belong to CCCC boundary condition.

- By enhancing the velocity of moving load, the transverse displacement and stresses will be increased.

- The maximum difference between linear and nonlinear transient deflection is approximately $100 \%$ for thin plates.

- By increasing the material gradient index, the amount of stresses and deflection considerably decreases.

- The amplitude of nonlinear deflection and stresses is smaller than the linear one.

- The differences between linear and nonlinear amounts for bottom of plate is larger than the top of plate. The most difference between linear and nonlinear values for stresses is approximately $50 \%$.

For the future analyses, higher order plate theories and application of Green geometric nonlinearity rather than Von Karman will be suggested, and also the behavior of 

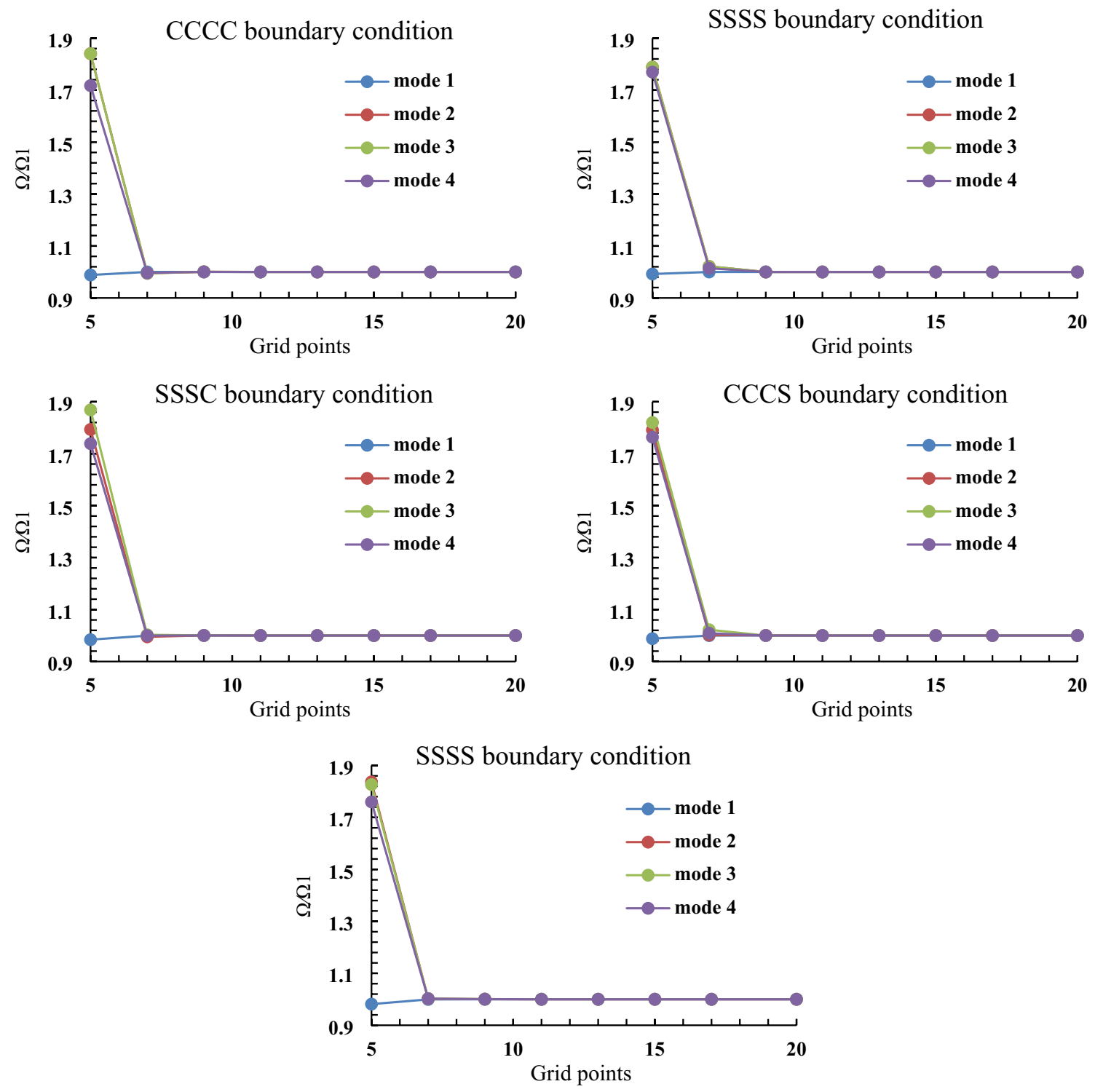

Fig. 6 convergence study of present model for different boundary condition 
Fig. 7 The effect of velocity of moving force on deflection history of center-point of the plate (CCCS boundary condition)

Fig. 8 The effect of velocity of moving force on deflection history of center-point of the plate (SSSS boundary condition)
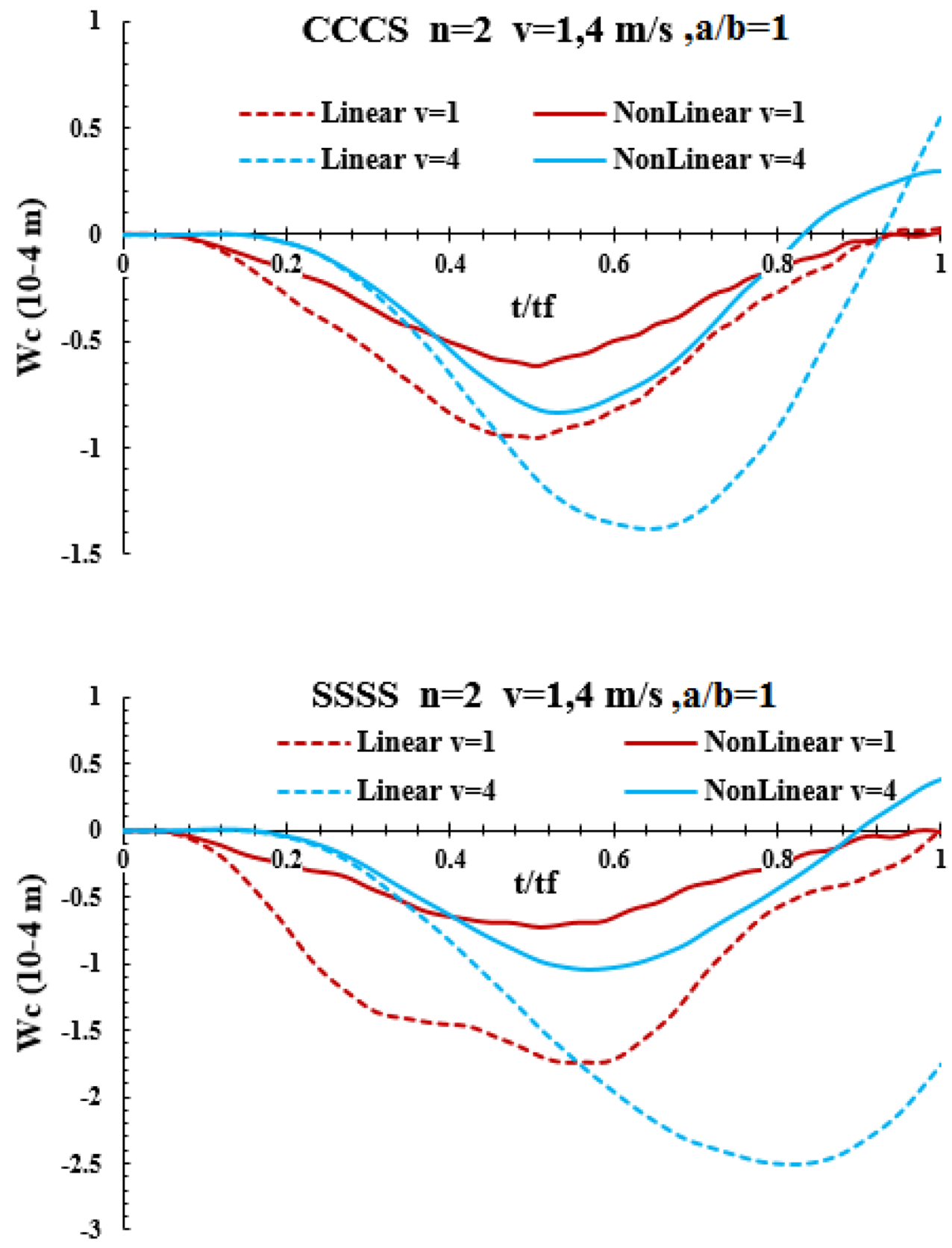
Fig. 9 The effect of velocity of moving force on deflection history of center-point of the plate (CCCC boundary condition)

Fig. 10 The effect of velocity of moving force on deflection history of center-point of the plate (SSSC boundary condition)
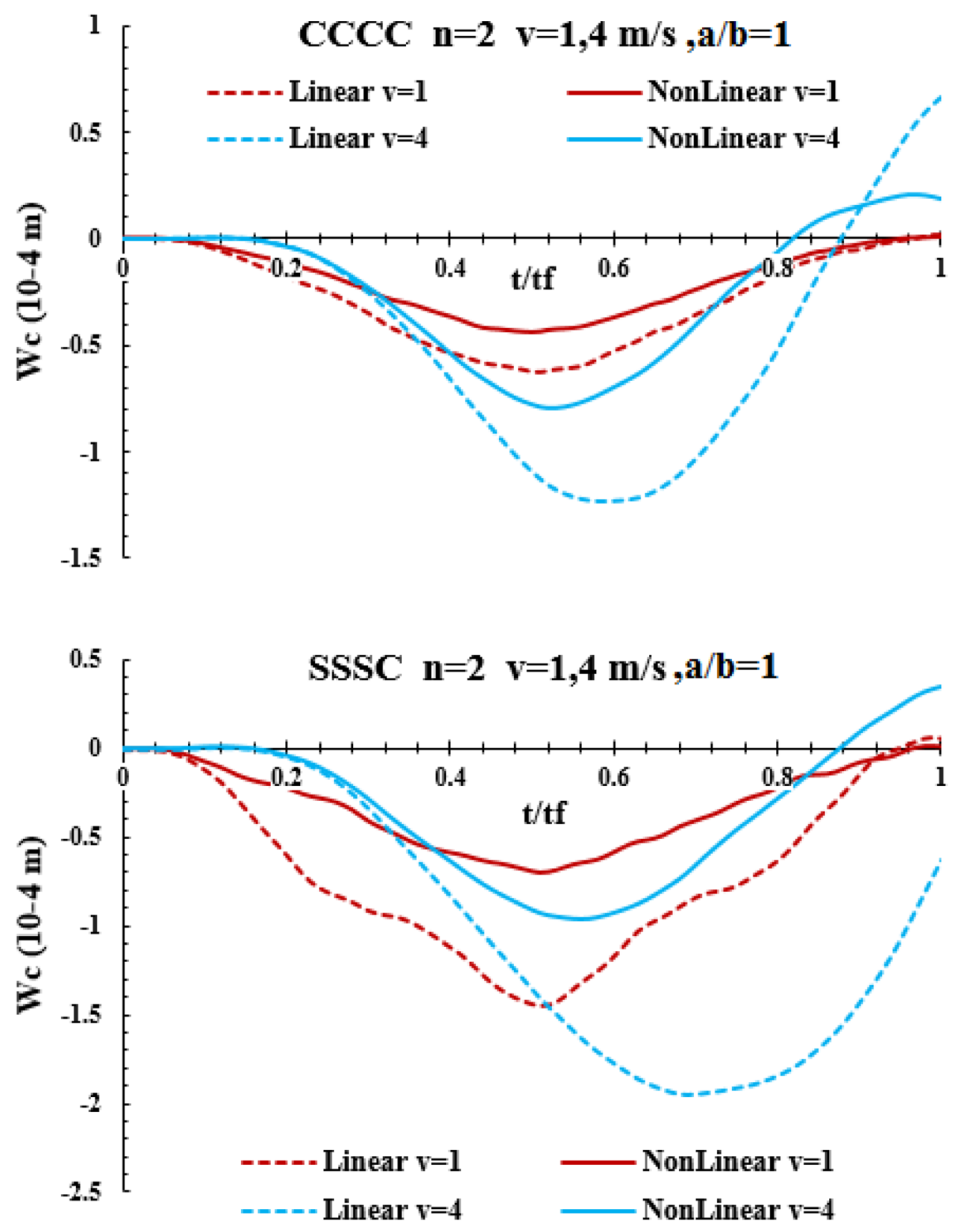
Fig. 11 The effect of velocity of moving force on deflection history of center-point of the plate (SSCC boundary condition)

Fig. 12 The effect of material gradient index on deflection history of center-point of the plate (SSSS boundary condition)
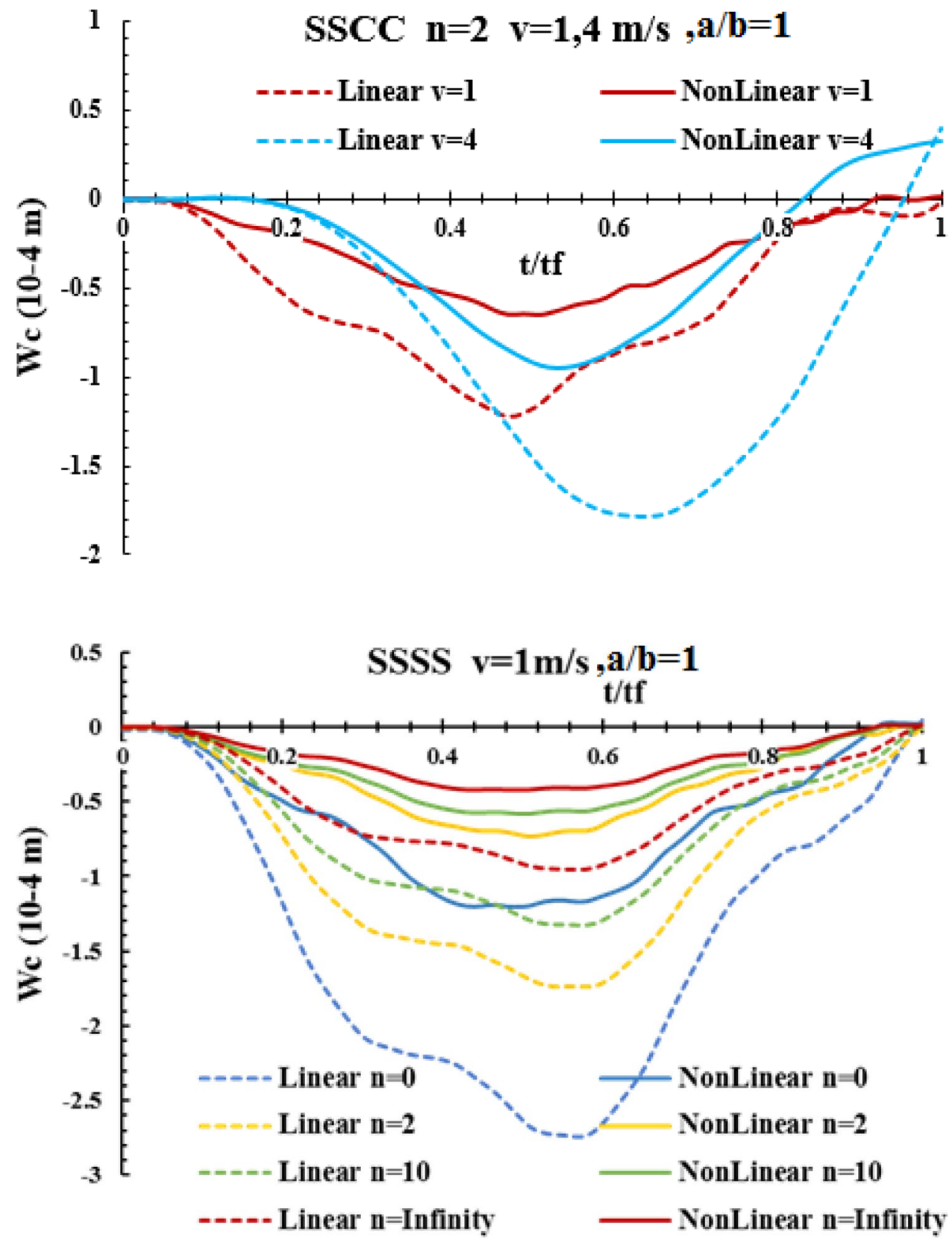
Fig. 13 The effect of material gradient index on deflection history of center-point of the plate (CCCC boundary condition)

Fig. 14 The effect of magnitude of moving load on deflection history of center-point of the plate (SSSS boundary condition)
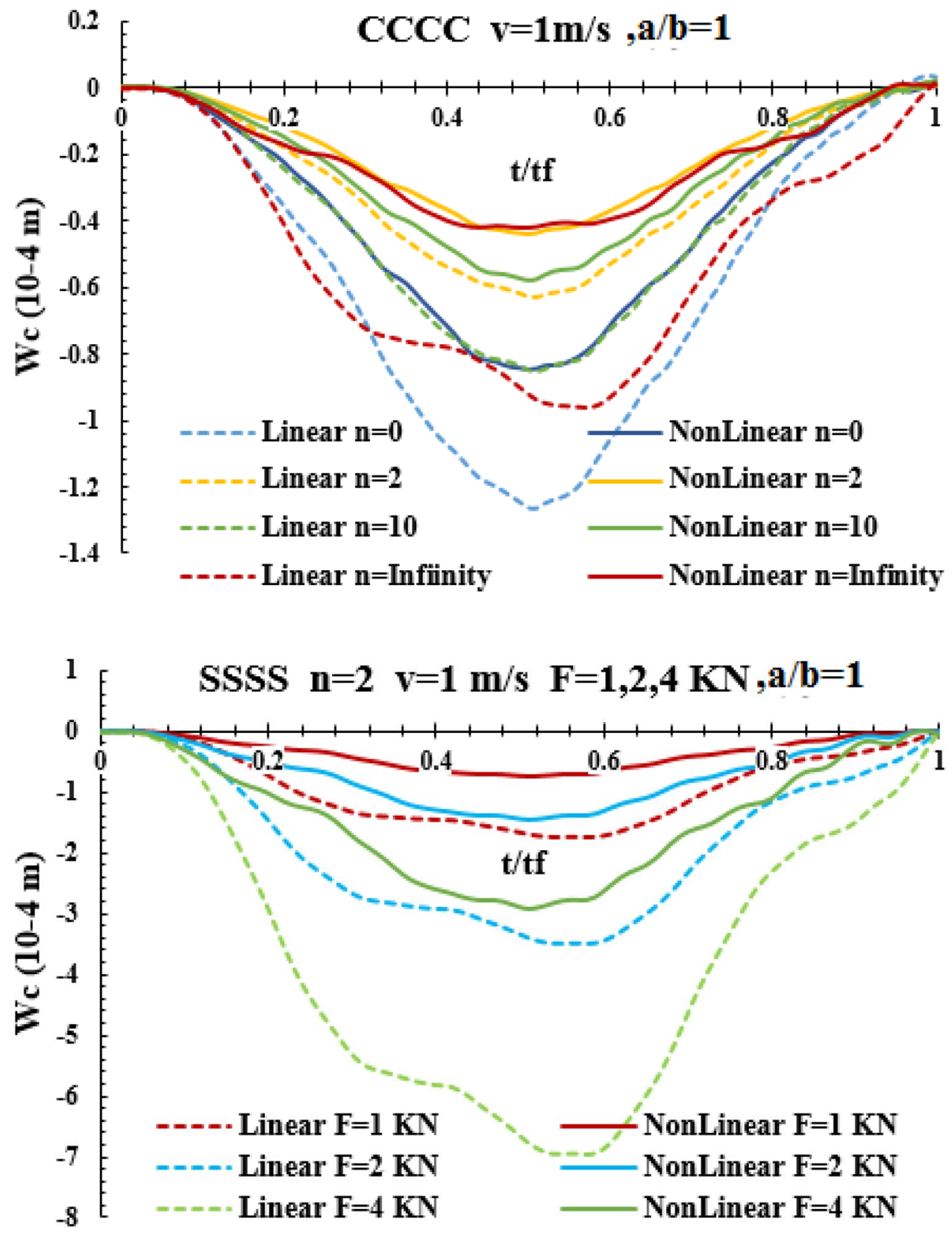
Fig. 15 The effect of magnitude of moving load on deflection history of center-point of the plate (SSSS boundary condition)

Fig. 16 The effect of aspect ratio on transient deflection of center-point of the plate (SSSS boundary condition)
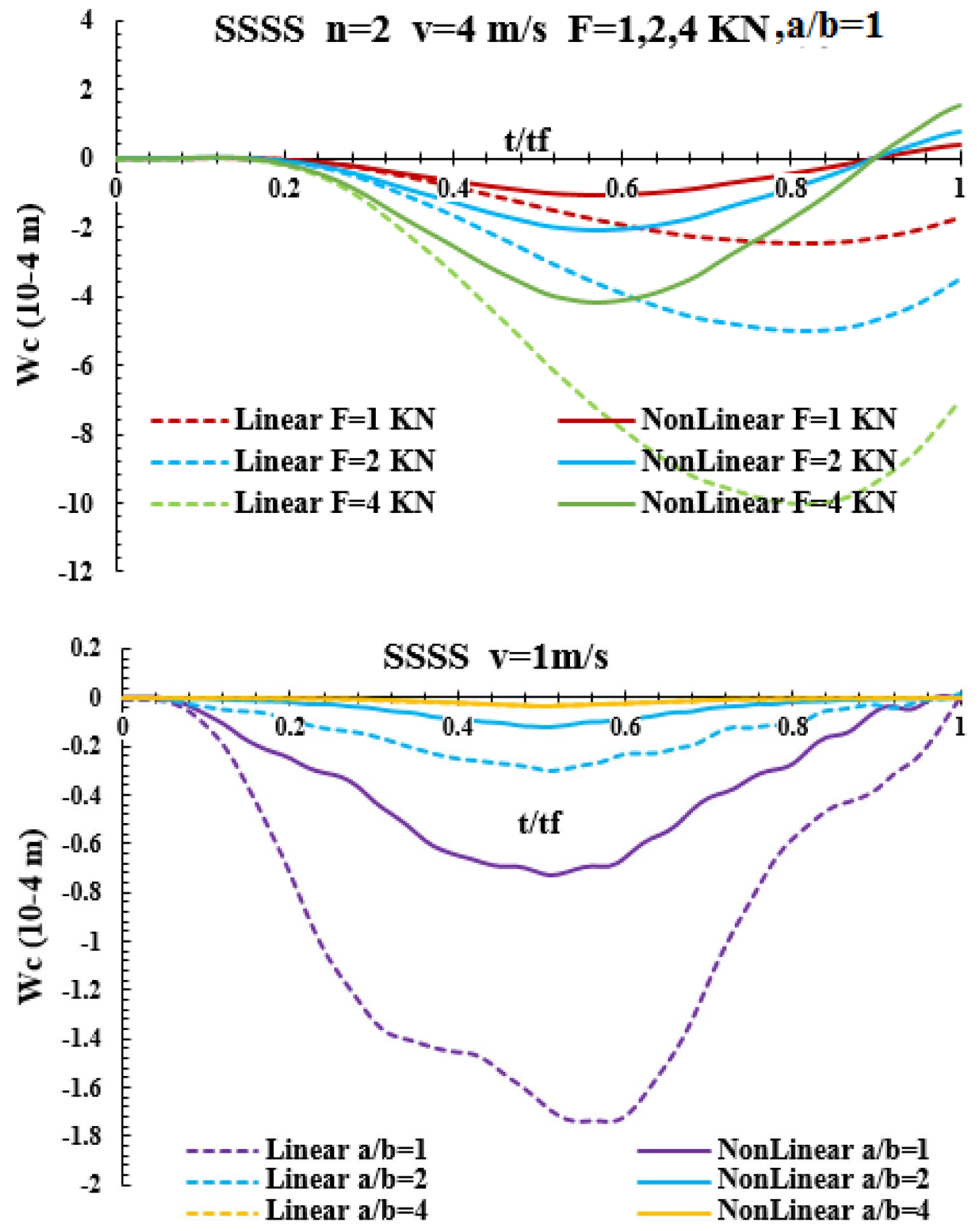
Fig. 17 The effect of aspect ratio on transient deflection of center-point of the plate (SSSS boundary condition)

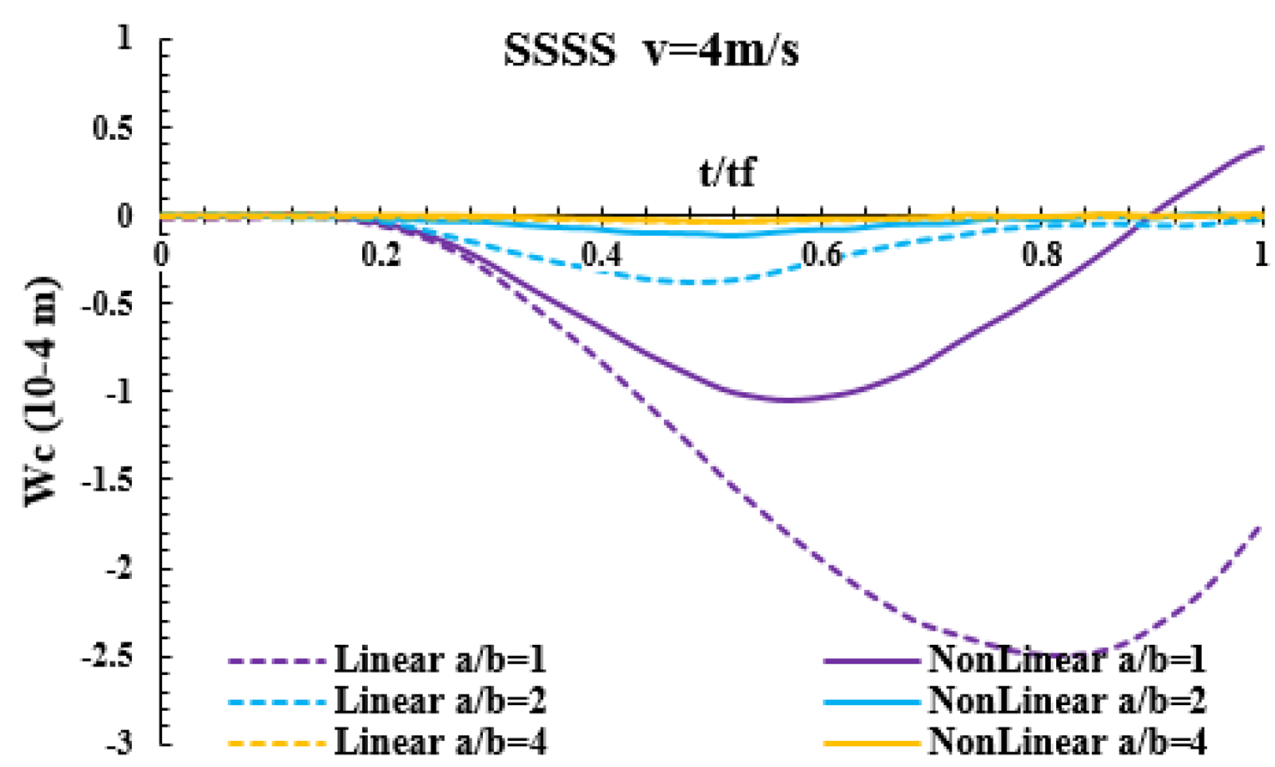

Fig. 18 The effect of material gradient index on transient distribution of $\sigma_{x x}$ for center-point of the plate (CCCC, $v=1 \mathrm{~m} / \mathrm{s}$ )

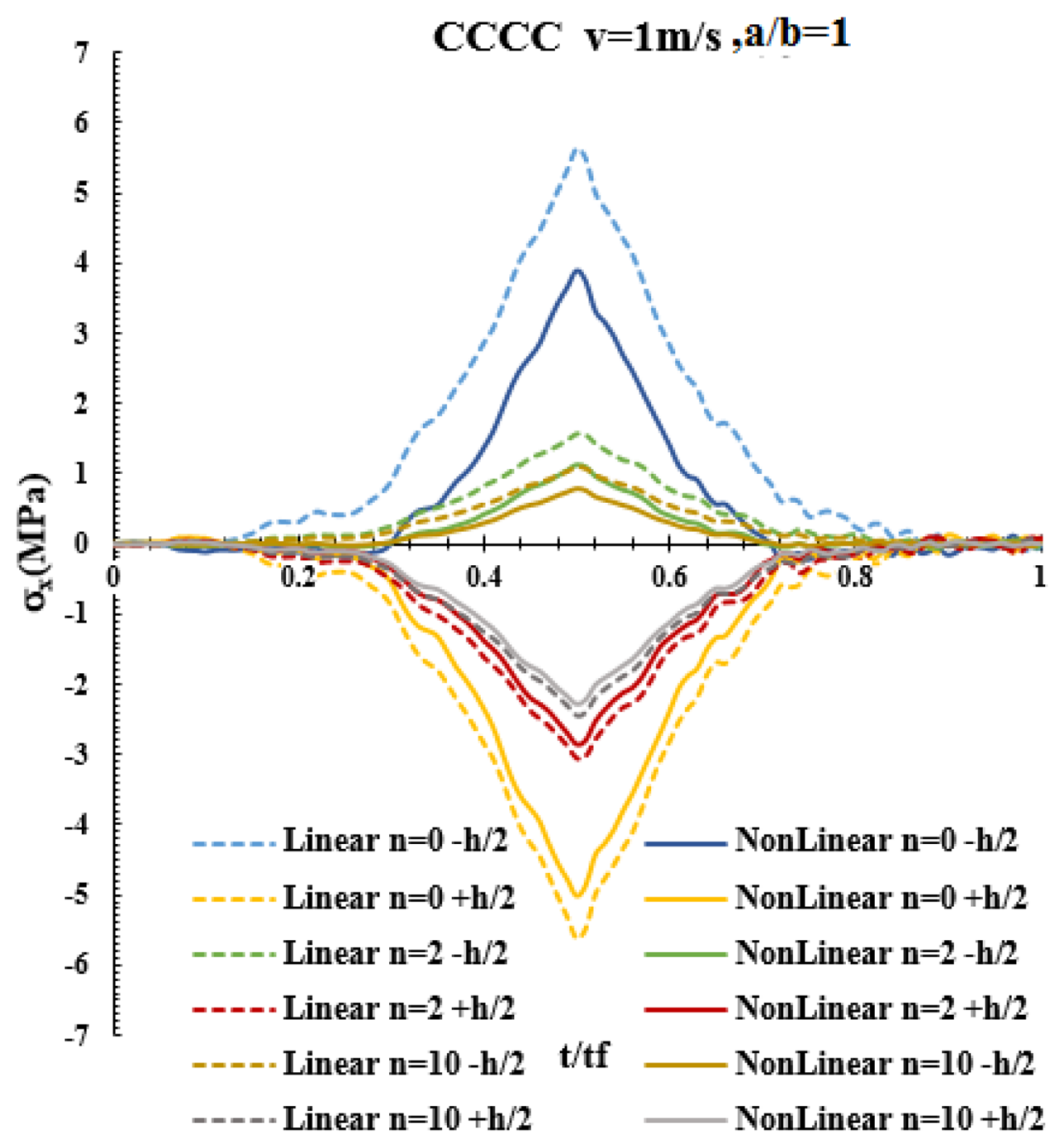


Fig. 19 The effect of material gradient index on transient distribution of $\sigma_{x x}$ for center-point of the plate (CCCC, $v=4 \mathrm{~m} / \mathrm{s}$ )

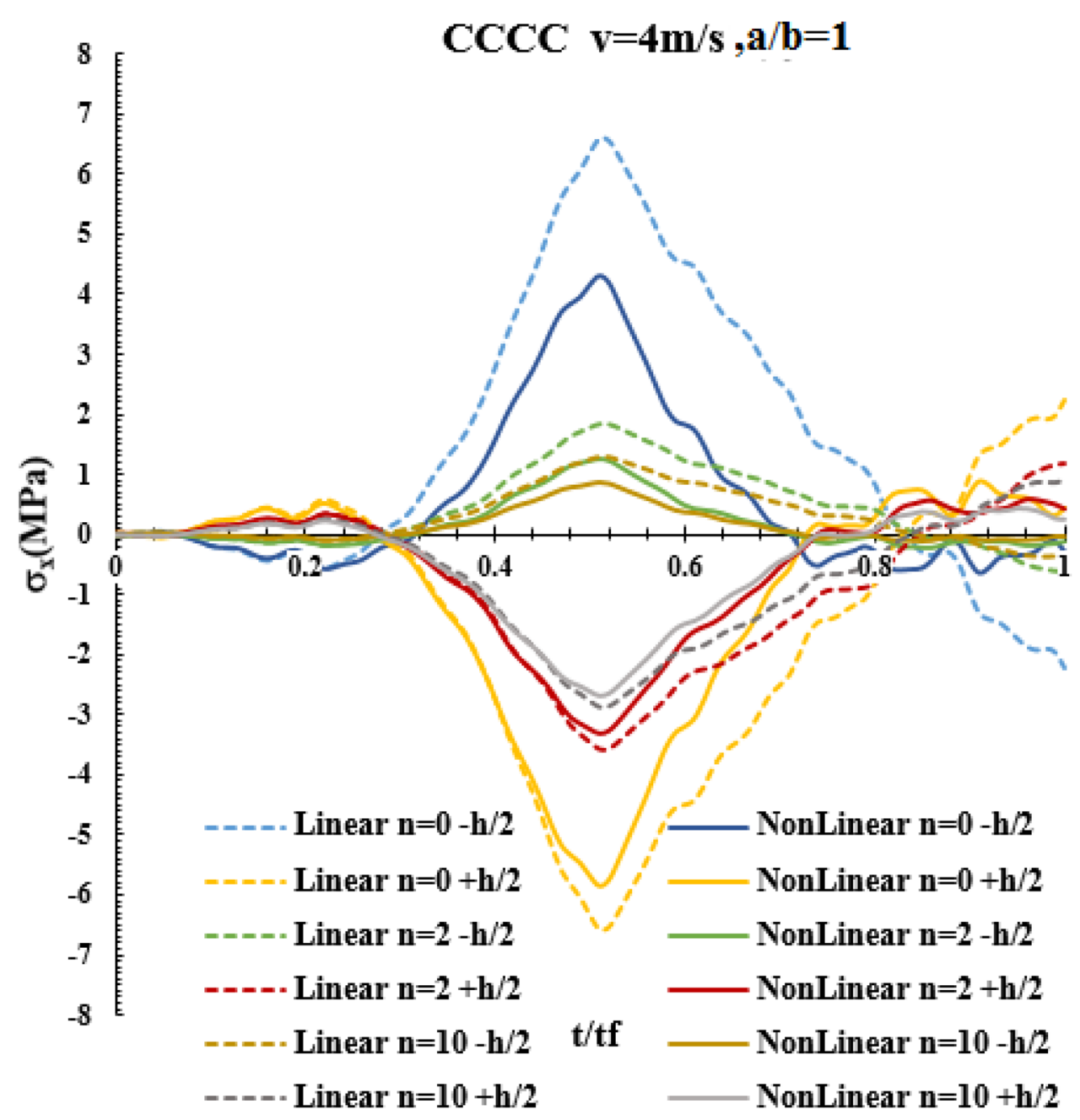


Fig. 20 The effect of material gradient index on transient distribution of $\sigma_{x x}$ for center-point of the plate (SSSS, $\mathrm{v}=1 \mathrm{~m} / \mathrm{s}$ )

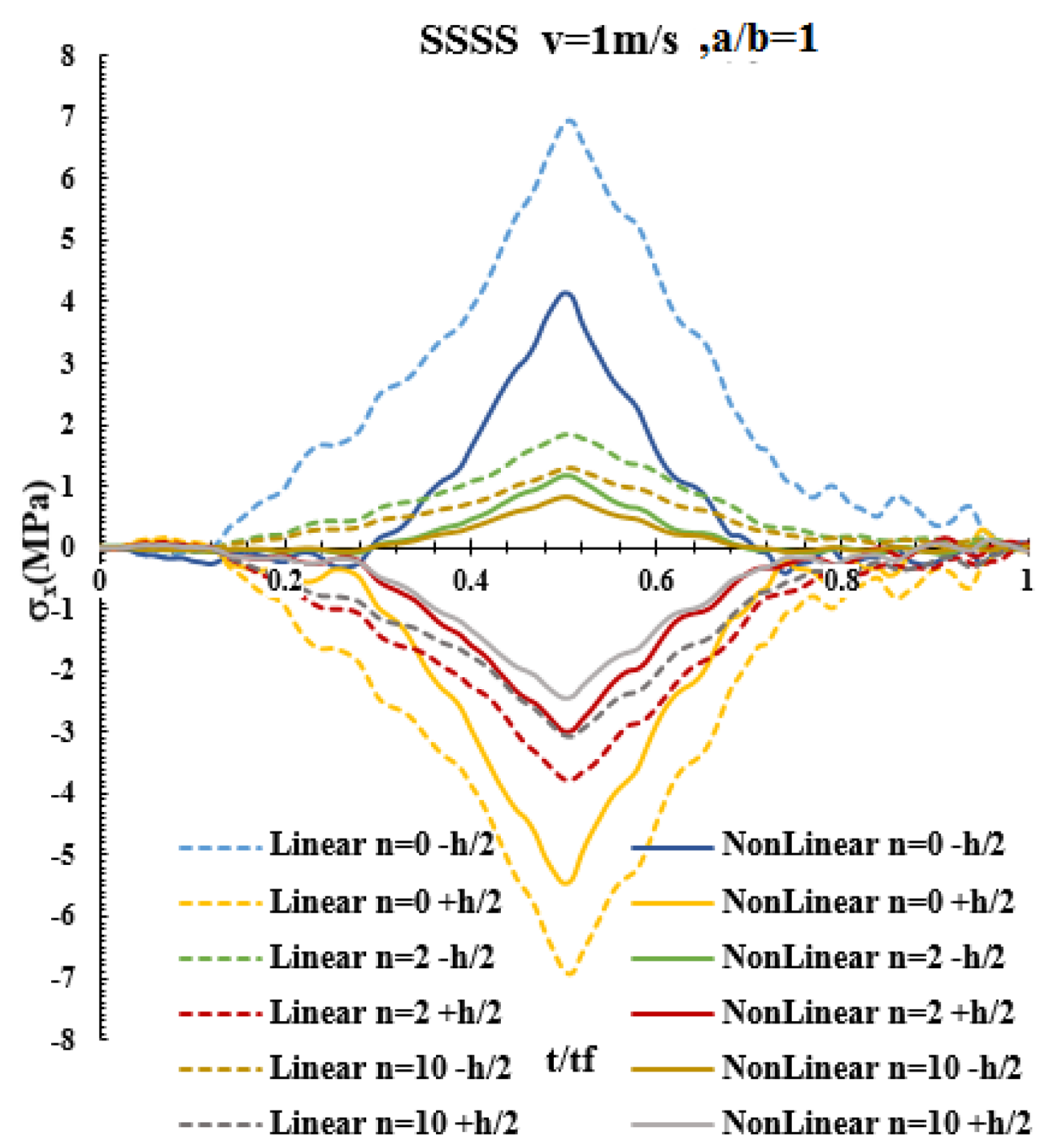


Fig. 21 The effect of material gradient index on transient distribution of $\sigma_{x x}$ for center-point of the plate (SSSS, $\mathrm{v}=1 \mathrm{~m} / \mathrm{s}$ )

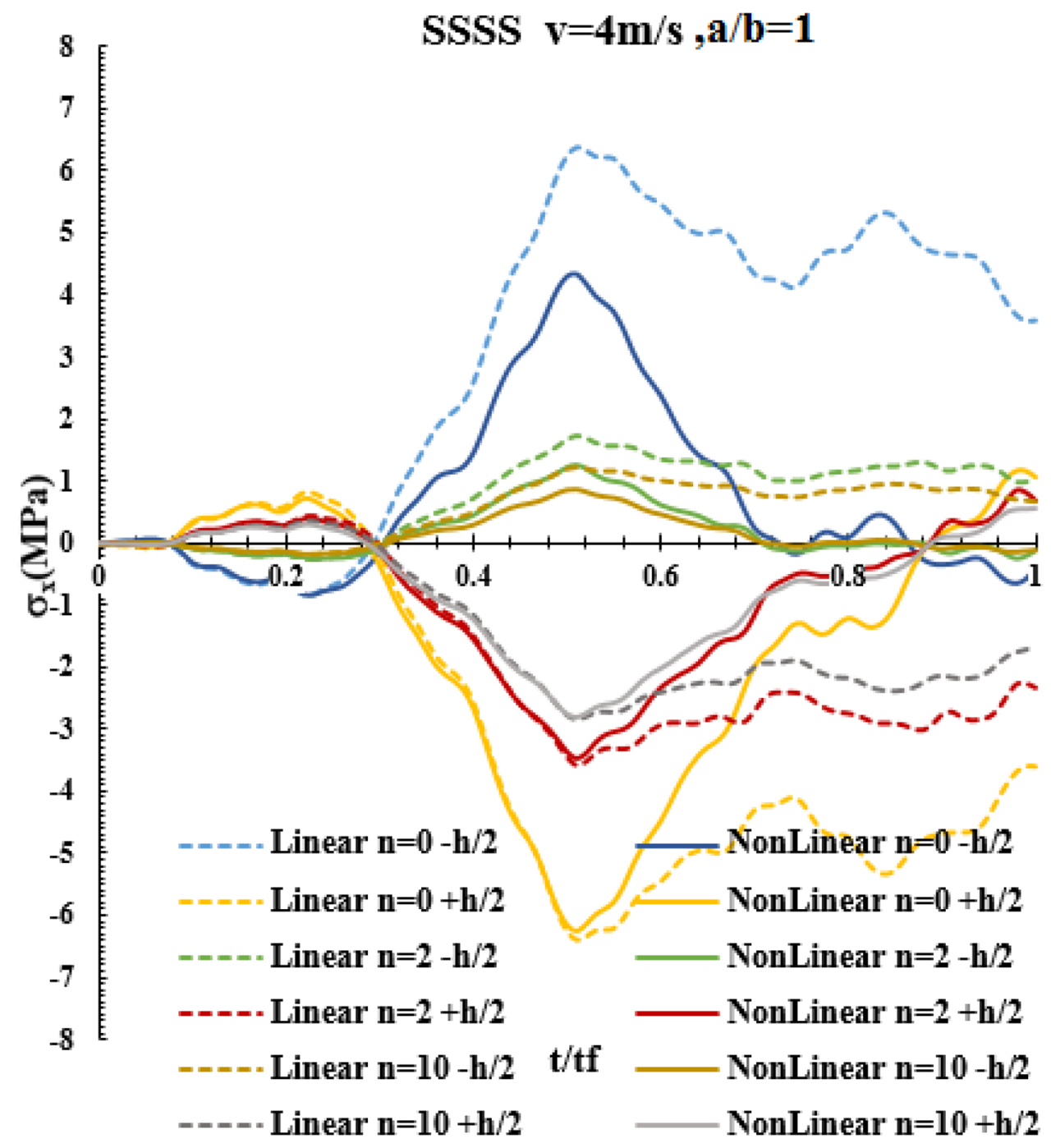


Fig. 22 The effect of material gradient index on transient distribution of $\sigma_{y y}$ for center-point of the plate (CCCC, $v=1 \mathrm{~m} / \mathrm{s}$ )

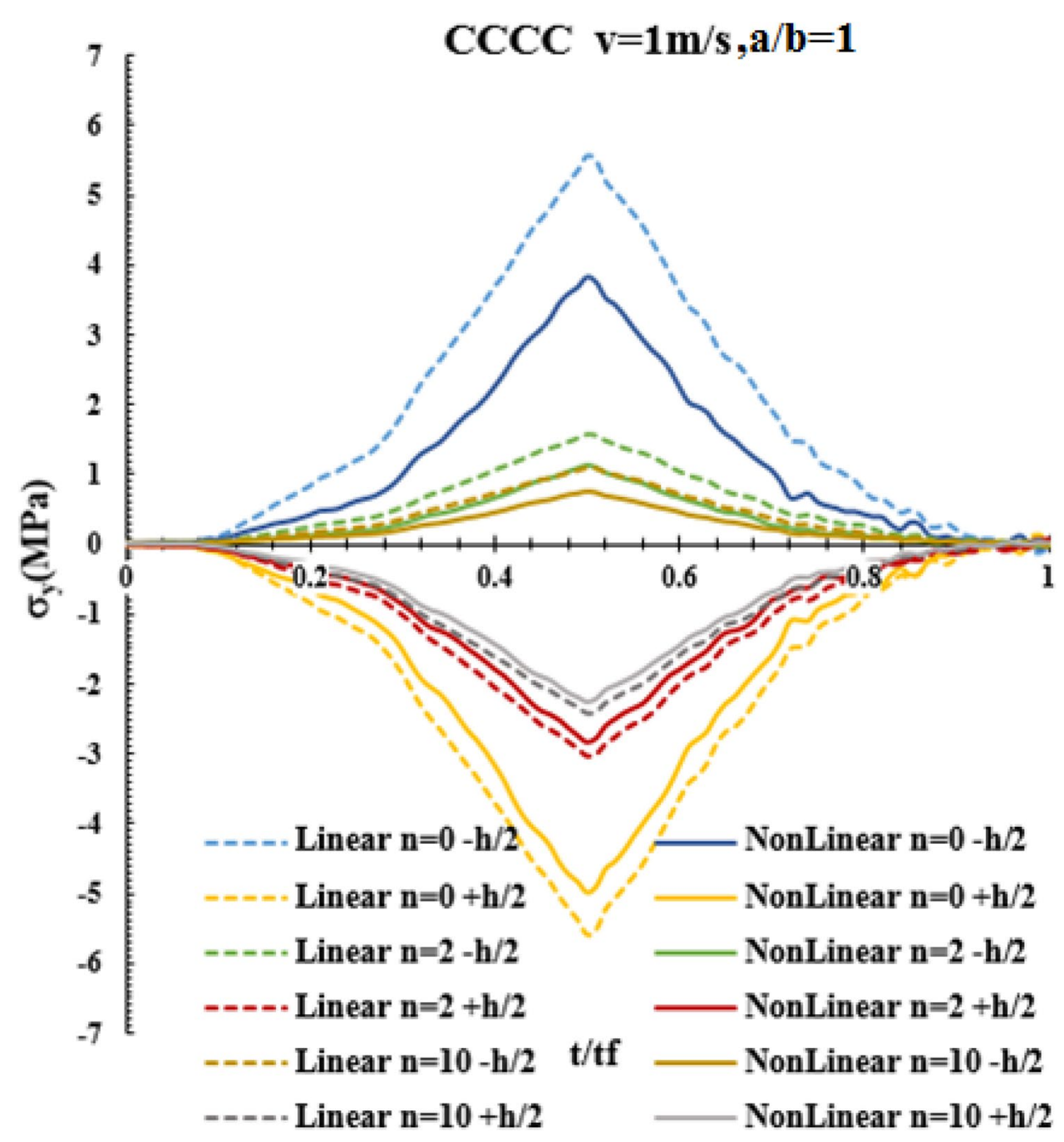


Fig. 23 The effect of material gradient index on transient distribution of $\sigma_{y y}$ for center-point of the plate (CCCC, $v=4 \mathrm{~m} / \mathrm{s}$ )

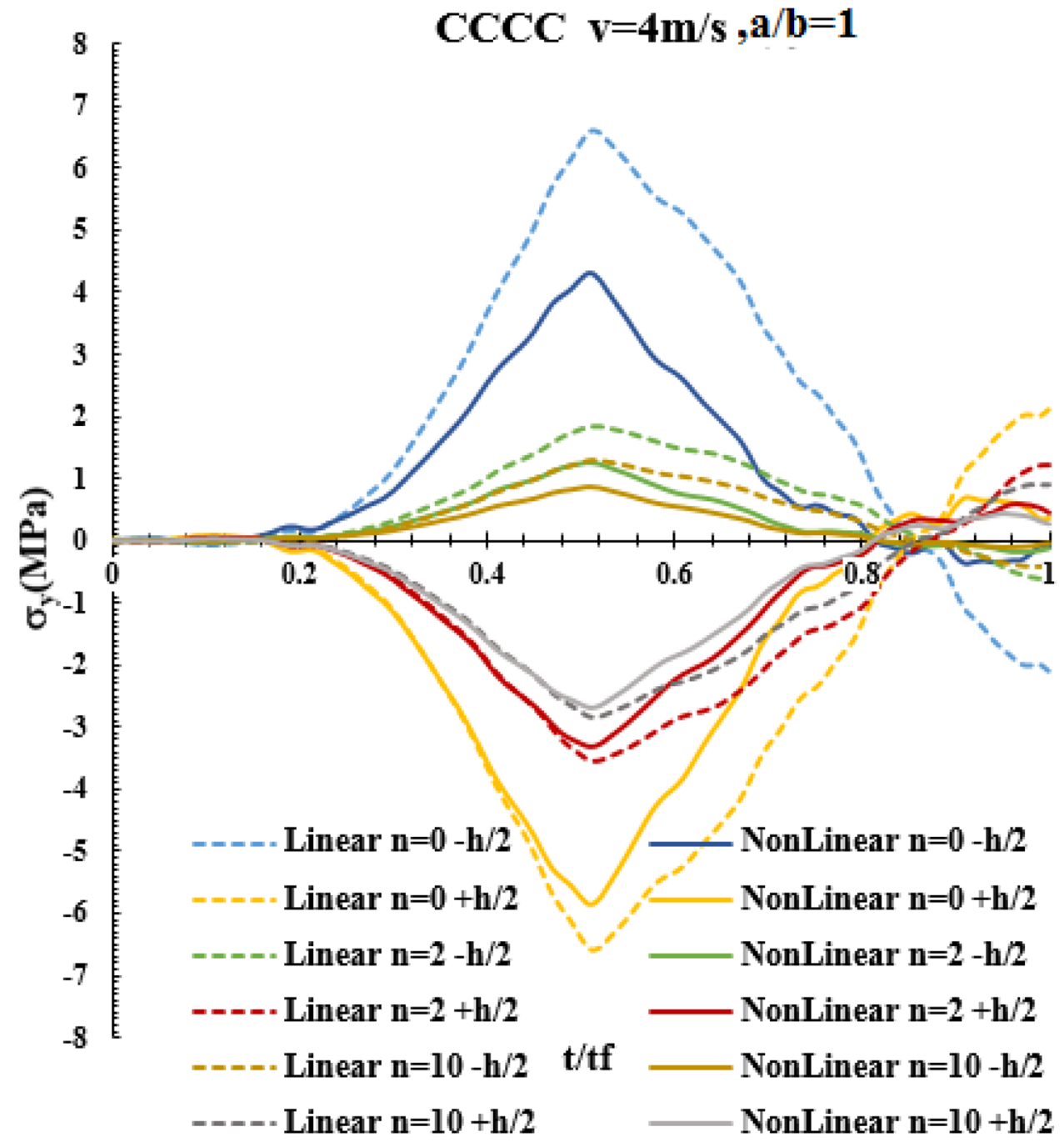


Fig. 24 the effect of material gradient index on transient distribution of $\sigma_{y y}$ for center-point of the plate (SSSS, $v=1 \mathrm{~m} / \mathrm{s}$ )

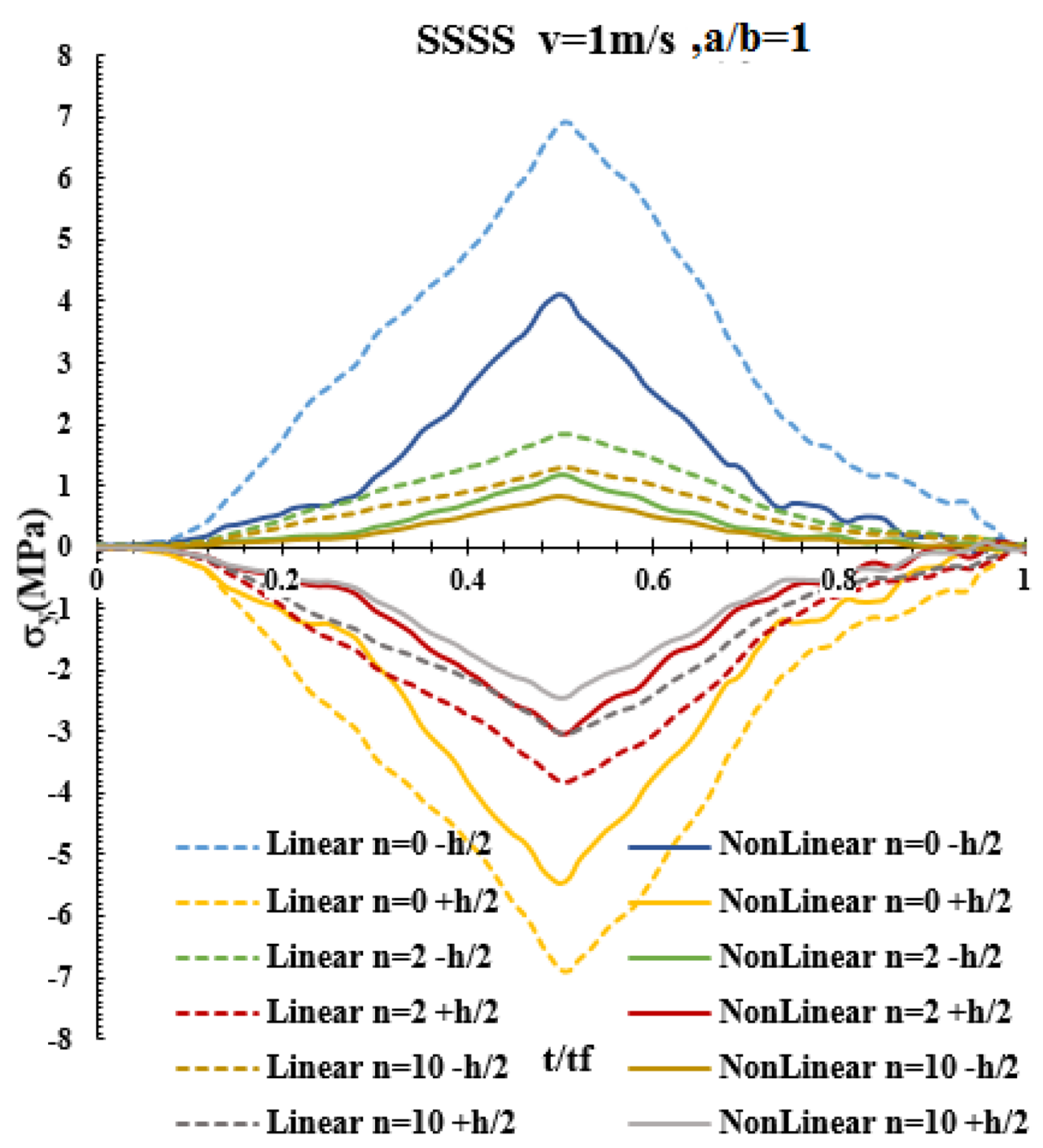


Fig. 25 the effect of material gradient index on transient distribution of $\sigma_{y y}$ for center-point of the plate (SSSS, $v=4 \mathrm{~m} / \mathrm{s}$ )

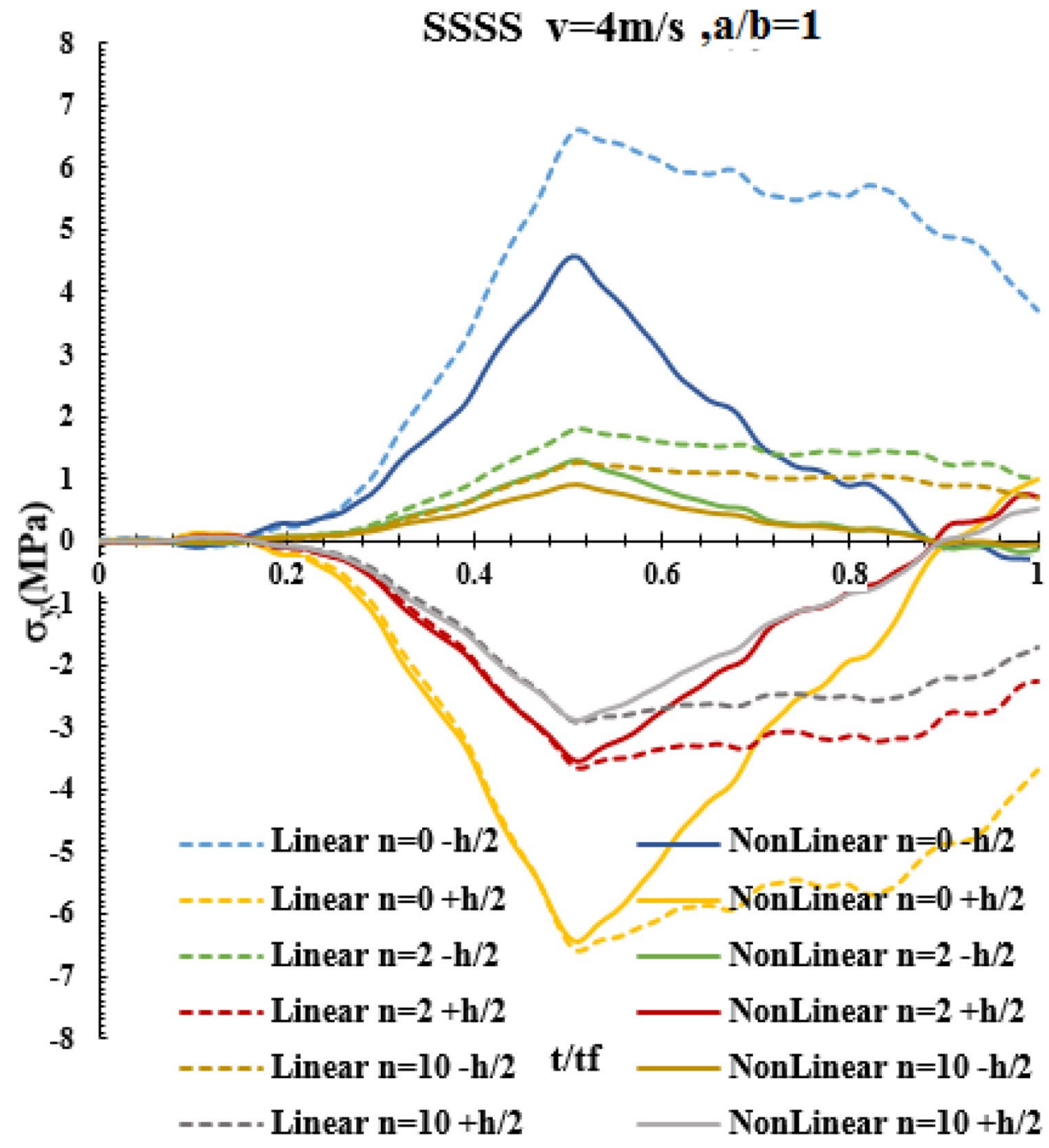

plates reinforced with graphene platelet (GPL) or carbon nanotube (CNT) may be investigated.

\section{Declarations}

Conflict of interest The authors declare that they have no conflict of interest.

Open Access This article is licensed under a Creative Commons Attribution 4.0 International License, which permits use, sharing, adaptation, distribution and reproduction in any medium or format, as long as you give appropriate credit to the original author(s) and the source, provide a link to the Creative Commons licence, and indicate if changes were made. The images or other third party material in this article are included in the article's Creative Commons licence, unless indicated otherwise in a credit line to the material. If material is not included in the article's Creative Commons licence and your intended use is not permitted by statutory regulation or exceeds the permitted use, you will need to obtain permission directly from the copyright holder. To view a copy of this licence, visit http://creativecommons. org/licenses/by/4.0/.

\section{References}

1. Hadipour M, Ahmadian MT, Lashkari SG, Barari A (2011) Natural frequency improvement of a suspended FGM Bridge. ASME Int Mech Eng Congr Expos 54945:699-704

2. Babaei M, Asemi K, Kiarasi F (2020) Static response and freevibration analysis of a functionally graded annular elliptical sector plate made of saturated porous material based on 3D finite element method. Mech Based Des Struct. https://doi.org/ 10.1080/15397734.2020.1864401

3. Mahamood RM, Akinlabi ET (2017) Types of functionally graded materials and their areas of application. In: Functionally graded materials. topics in mining, metallurgy and materials engineering. Springer, Cham. https://doi.org/10.1007/ 978-3-319-53756-6_2

4. Babaei M, Asemi K, Kiarasi F (2021) Dynamic analysis of functionally graded rotating thick truncated cone made of saturated porous materials. Thin-Walled Struct 164:107852

5. Shahsavari M, Asemi K, Babaei M, Kiarasi F (2021) Numerical investigation on thermal post-buckling of annular sector plates made of FGM via 3D finite element method. Mech Adv Compos Struct. https://doi.org/10.22075/macs.2021.21158.1293 
6. Yang J, Chen Y, Xiang Y, Jia XL (2008) Free and forced vibration of cracked inhomogeneous beams under an axial force and a moving load. J Sound Vib 312(1-2):166-181

7. Malekzadeh P, Monajjemzadeh SM (2013) Dynamic response of functionally graded plates in thermal environment under moving load. Compos B Eng 45(1):1521-1533

8. Malekzadeh P, Monajjemzadeh SM (2015) Nonlinear response of functionally graded plates under moving load. Thin-Walled Struct 96:120-129

9. Sofiyev AH (2010) Dynamic response of an FGM cylindrical shell under moving loads. Compos Struct 93(1):58-66

10. Chen Y, Fu Y, Zhong J, Li Y (2017) Nonlinear dynamic responses of functionally graded tubes subjected to moving load based on a refined beam model. Nonlinear Dyn 88(2):1441-1452

11. Yas MH, Heshmati M (2012) Dynamic analysis of functionally graded nanocomposite beams reinforced by randomly oriented carbon nanotube under the action of moving load. Appl Math Model 36(4):1371-1394

12. Şimşek $M$ (2010) Non-linear vibration analysis of a functionally graded Timoshenko beam under action of a moving harmonic load. Compos Struct 92(10):2532-2546

13. Ebrahimzadeh Hassanabadi M, Vaseghi Amiri J, Davoodi MR (2014) On the vibration of a thin rectangular plate carrying a moving oscillator. Sci Iran 21(2):284-294

14. Esen I (2015) A new FEM procedure for transverse and longitudinal vibration analysis of thin rectangular plates subjected to a variable velocity moving load along an arbitrary trajectory. Latin Am J Solids Struct 12(4):808-830

15. Assie $A, A$ kbaş ŞD, Bashiri AH, Abdelrahman AA, Eltaher MA (2021) Vibration response of perforated thick beam under moving load. Eur Phys J Plus 136(3):1-15

16. Uzzal RUA, Bhat RB, Ahmed W (2012) Dynamic response of a beam subjected to moving load and moving mass supported by Pasternak foundation. Shock Vib 19(2):201-216

17. Nguyen DK, Nguyen QH, Tran TT (2017) Vibration of bi-dimensional functionally graded Timoshenko beams excited by a moving load. Acta Mech 228(1):141-155

18. Kiani Y (2017) Dynamics of FG-CNT reinforced composite cylindrical panel subjected to moving load. Thin-Walled Struct 111:48-57

19. Kiani Y (2017) Analysis of FG-CNT reinforced composite conical panel subjected to moving load using Ritz method. Thin-Walled Struct 119:47-57

20. Kiani Y (2020) Influence of graphene platelets on the response of composite plates subjected to a moving load. Mech Based Des Struct. https://doi.org/10.1080/15397734.2020.1744006

21. Sorrentino S, Catania G (2018) Dynamic analysis of rectangular plates crossed by distributed moving loads. Math Mech Solids 23(9):1291-1302

22. Shirmohammadi F, Bahrami S, et Saadatpour MM (2017) Dynamic response of rectangular plate subjected to moving loads using spectral finite strip method.

23. Eyvazian A, Shahsavari D, Karami B (2020) On the dynamic of graphene reinforced nanocomposite cylindrical shells subjected to a moving harmonic load. Int J Eng Sci 154:103339

24. Luong VH, Cao TNT, Lieu QX, Nguyen XV (2020) Moving element method for dynamic analyses of functionally graded plates resting on Pasternak foundation subjected to moving harmonic load. Int J Struct Stab Dyn 20(01):2050003

25. Esmaeilzadeh M, Kadkhodayan M (2019) Dynamic analysis of stiffened bi-directional functionally graded plates with porosities under a moving load by dynamic relaxation method with kinetic damping. Aerosp Sci Technol 93:105333

26. Akgün G, Kurtaran H, Kalbaran Ö (2021) Non-linear transient response of porous functionally graded truncated conical panels using GDQ method. Thin-Walled Struct 159:107276
27. Akgün G, Kurtaran H (2019) Large displacement transient analysis of FGM super-elliptic shells using GDQ method. Thin-Walled Struct 141:133-152

28. Duc ND, Cong PH, Quang VD (2016) Nonlinear dynamic and vibration analysis of piezoelectric eccentrically stiffened FGM plates in thermal environment. Int J Mech Sci 115:711-722

29. Duc ND, Quan TQ (2014) Transient responses of functionally graded double curved shallow shells with temperature-dependent material properties in thermal environment. Eur J Mech-A/ Solids 47:101-123

30. Nguyen DD, Tran QQ, Nguyen DK (2017) New approach to investigate nonlinear dynamic response and vibration of imperfect functionally graded carbon nanotube reinforced composite double curved shallow shells subjected to blast load and temperature. Aerosp Sci Technol 71:360-372

31. Quan TQ, Tran P, Tuan ND, Duc ND (2015) Nonlinear dynamic analysis and vibration of shear deformable eccentrically stiffened S-FGM cylindrical panels with metal-ceramic-metal layers resting on elastic foundations. Compos Struct 126:16-33

32. Duc ND (2014) Nonlinear static and dynamic stability of functionally graded plates and shells. Vietnam: Vietnam Natl Univ Press. Google Scholar.

33. Cong PH, Khanh ND, Khoa ND, Duc ND (2018) New approach to investigate nonlinear dynamic response of sandwich auxetic double curves shallow shells using TSDT. Compos Struct 185:455-465

34. Kiarasi F, Babaei M, Dimitri R, Tornabene F (2021) Hygrothermal modeling of the buckling behavior of sandwich plates with nanocomposite face sheets resting on a Pasternak foundation. Continuum Mech Thermodyn 33:911-932

35. Asemi K, Babaei M, Kiarasi F (2020) Static, natural frequency and dynamic analyses of functionally graded porous annular sector plates reinforced by graphene platelets. Mech Based Des Struct. https://doi.org/10.1080/15397734.2020.1822865

36. Wang $X$ (2015) Differential quadrature and differential quadrature based element methods: theory and applications. Butterworth-Heinemann

37. Shu C (2012) Differential quadrature and its application in engineering. Springer, Berlin

38. Bellman R, Kashef BG, Casti J (1972) Differential quadrature: a technique for the rapid solution of nonlinear partial differential equations. J Comput Phys 10(1):40-52

39. Babaei M, Hajmohammad MH, Asemi K (2020) Natural frequency and dynamic analyses of functionally graded saturated porous annular sector plate and cylindrical panel based on 3D elasticity. Aerosp Sci Technol 96:105524

40. Babaei M, Asemi K, Safarpour P (2019) Natural frequency and dynamic analyses of functionally graded saturated porous beam resting on viscoelastic foundation based on higher order beam theory. J Solid Mech 11(3):615-634

41. Babaei M, Asemi K (2020) Static, dynamic and natural frequency analyses of functionally graded carbon nanotube annular sector plates resting on viscoelastic foundation. SN Appl Sci 2(10):1-21

42. Hashemi SH, Arsanjani M (2005) Exact characteristic equations for some of classical boundary conditions of vibrating moderately thick rectangular plates. Int J Solids Struct 42(3-4):819-853

43. Rao S, Sheikh A, Mukhopadhyay M (1993) Large-amplitude finite element flexural vibration of plates/stiffened plates. J Acoust Soc Am 93(6):3250-3257

44. Eftekhari S, Jafari A (2012) A mixed method for free and forced vibration of rectangular plates. Appl Math Model 36(6):2814-2831

Publisher's Note Springer Nature remains neutral with regard to jurisdictional claims in published maps and institutional affiliations. 